\title{
Homogeneity of cohomology classes associated with Koszul matrix factorizations
}

\author{
Alexander Polishchuk
}

\begin{abstract}
In this work we prove the so called dimension property for the cohomological field theory associated with a homogeneous polynomial $W$ with an isolated singularity, in the algebraic framework of [36]. This amounts to showing that some cohomology classes on the Deligne-Mumford moduli spaces of stable curves, constructed using Fourier-Mukai type functors associated with matrix factorizations, live in prescribed dimension. The proof is based on a homogeneity result established in [33 for certain characteristic classes of Koszul matrix factorizations of 0 . To reduce to this result we use the theory of Fourier-Mukai type functors involving matrix factorizations and the natural rational lattices in the relevant Hochschild homology spaces, as well as a version of Hodge-Riemann bilinear relations for Hochschild homology of matrix factorizations. Our approach also gives a proof of the dimension property for the cohomological field theories associated with some quasihomogeneous polynomials with an isolated singularity.
\end{abstract}

2010 Mathematics Subject Classification. Primary 81T70; Secondary 14B05, 16E40 Keywords. Matrix factorization, Fan-Jarvis-Ruan-Witten theory, Hochschild homology

\section{Introduction}

\subsection{Dimension property in Fan-Jarvis-Ruan-Witten theory}

Let $W \in \mathbb{C}\left[x_{1}, \ldots, x_{n}\right]$ be a quasihomogeneous polynomial with an isolated singularity at the origin. Fan, Jarvis and Ruan introduced in [16] an analog of the Gromov-Witten theory associated with $W$ and with a finite subgroup $G \subset\left(\mathbb{C}^{*}\right)^{n}$ of diagonal symmetries of $W$ (such that $G$ contains the exponential grading operator $J$ associated with the weights of the variables $\left.x_{1}, \ldots, x_{n}\right)$. This theory, often referred to as Fan-Jarvis-Ruan-Witten theory (FJRW-theory), consists of a collection of maps

$$
\Lambda_{g, r}: H^{\otimes r} \rightarrow H^{*}\left(\bar{M}_{g, r}, \mathbb{C}\right)
$$

where $\bar{M}_{g, r}$ is the Deligne-Mumford compactification of the moduli spaces of curves with $r$ marked points, and $H=H_{W, G}$ is a finite-dimensional vector space associated with $(W, G)$ (called the space state of the theory). The maps $\Lambda_{g, r}$ satisfy some gluing axioms on the 
boundary components of $\bar{M}_{g, r}$, that constitute the notion of a cohomological field theory, introduced by Kontsevich and Manin [20]. In fact, the state space of the FJRW-theory has a decomposition

$$
H_{W, G}=\bigoplus_{\gamma \in G} H\left(W_{\gamma}\right)^{G}
$$

where $W_{\gamma}=\left.W\right|_{\left(\mathbb{A}^{n}\right)^{\gamma}}$, the restriction of $W$ to the space of $\gamma$-invariants, and

$$
H\left(W\left(x_{1}, \ldots, x_{n}\right)\right):=\left(\Omega_{\mathbb{A}^{n}}^{n} /\left(d W \wedge \Omega_{\mathbb{A}^{n}}^{n-1}\right)\right)
$$

(the latter definition is applied to all $W_{\gamma}$ ). Each component of the map $\Lambda_{g, r}$ factors as a composition

$$
H\left(W_{\gamma_{1}}\right)^{G} \otimes \ldots \otimes H\left(W_{\gamma_{r}}\right)^{G} \stackrel{\phi_{g}\left(\gamma_{1}, \ldots, \gamma_{r}\right)}{\longrightarrow} H^{*}\left(\mathcal{S}_{g, G}\left(\gamma_{1}, \ldots, \gamma_{r}\right), \mathbb{C}\right) \rightarrow H^{*}\left(\bar{M}_{g, r}, \mathbb{C}\right),
$$

where $\mathcal{S}_{g, G}\left(\gamma_{1}, \ldots, \gamma_{r}\right) \rightarrow \bar{M}_{g, r}$ is some finite covering, corresponding to choices of generalized spin-structure (of type $\gamma_{1}, \ldots, \gamma_{r}$ ) on a curve. For details, see [16] and [36].

Whereas in the original approach of [16] the maps $\phi_{g}\left(\gamma_{1}, \ldots, \gamma_{r}\right)$ were defined by studying a certain PDE (Witten's equation), in [36] we constructed these maps using Hochschild homology and the categories of matrix factorizations. More precisely, we use natural embeddings

$$
H(W)^{G} \subset H H_{*}\left(\mathrm{MF}_{G}(W)\right)
$$

where $\mathrm{MF}_{G}(W)$ is the category of $G$-equivariant matrix factorizations of $W$, and construct the maps $\phi_{g}\left(\gamma_{1}, \ldots, \gamma_{r}\right)$ as maps induced on Hochschild homology by some Fourier-Mukai type functor

$$
\operatorname{MF}_{G}\left(W_{\gamma_{1}} \oplus \ldots \oplus W_{\gamma_{r}}\right) \rightarrow D^{b}\left(\mathcal{S}_{g, G}\left(\gamma_{1}, \ldots, \gamma_{r}\right)\right),
$$

where $D^{b}(X)$ denotes the derived category of coherent sheaves on $X$.

Conjecturally, the algebraic approach of [36] produces the same theory as in [16], however, this is currently known to be true only for simple singularities (see [36, Sec. 7]), in the socalled narrow sectors (see [9]), and for most invertible polynomials and the maximal groups of symmetries (see [17]). In general, the hope is that the algebraic approach will be more accessible for calculations (as for example, the work [17] indicates), so it is important to establish algebraically all the properties of the FJRW-theory.

One of the properties of the maps $\phi_{g}\left(\gamma_{1}, \ldots, \gamma_{r}\right)$ which arises naturally in the analytic approach of [16] is the dimension property stating that

$$
\operatorname{im}\left(\phi_{g}\left(\gamma_{1}, \ldots, \gamma_{r}\right)\right) \subset H^{2 D_{g}\left(\gamma_{1}, \ldots, \gamma_{r}\right)+n_{1}+\ldots+n_{r}}\left(\mathcal{S}_{g, G}\left(\gamma_{1}, \ldots, \gamma_{r}\right), \mathbb{C}\right),
$$

with $n_{i}=\operatorname{dim}\left(\mathbb{A}^{N}\right)^{\gamma_{i}}$ and

$$
D_{g}\left(\gamma_{1}, \ldots, \gamma_{r}\right)=(g-1) \hat{c}+\iota_{\gamma_{1}}+\ldots+\iota_{\gamma_{r}}=-\sum_{j=1}^{n} \chi\left(C, L_{j}\right),
$$

where $\left(C, L_{1}, \ldots, L_{n}\right)$ is a smooth curve with a generalized spin-structure from the moduli space $\mathcal{S}_{g, G}\left(\gamma_{1}, \ldots, \gamma_{r}\right)$ and the numbers $\hat{c}=\hat{c}_{W}, \iota_{\gamma_{1}}, \ldots, \iota_{\gamma_{r}}$ are determined using the weights of the variables $x_{1}, \ldots, x_{n}$ (see [16, Sec. 3.2]). 
This property is not at all clear in the algebraic framework of [36]. The goal of this paper is to prove the dimension property in this framework assuming that $W\left(x_{1}, \ldots, x_{n}\right)$ is a homogeneous polynomial, i.e., the degrees of the variables are $\operatorname{deg}\left(x_{1}\right)=\ldots=\operatorname{deg}\left(x_{n}\right)=1$.

More generally, for a quasihomogeneous polynomial $W\left(x_{1}, \ldots, x_{n}\right)$, where $\operatorname{deg}\left(x_{i}\right)=d_{i}>$ 0 , we can define a homogeneous polynomial

$$
\widetilde{W}\left(y_{1}, \ldots, y_{n}\right)=W\left(y_{1}^{d_{1}}, \ldots, y_{n}^{d_{n}}\right)
$$

in new variables $y_{1}, \ldots, y_{n}$ with $\operatorname{deg}\left(y_{i}\right)=1$. We will prove the dimension property for the algebraic cohomological field theory associated with $(W, G)$ (for any $G$ ) provided $\widetilde{W}$ still has an isolated singularity at 0 .

Theorem 1.1.1. Let $W\left(x_{1}, \ldots, x_{n}\right)$ be a quasihomogeneous polynomial with an isolated singularity, $G$ a finite group of diagonal symmetries of $W$, containing the exponential grading element. Assume that $\widetilde{W}$ still has an isolated singularity at 0 . Then the maps $\phi_{g}\left(\gamma_{1}, \ldots, \gamma_{r}\right)$ defined in [36] satisfy the dimension property (1.2).

This will be deduced from a more general Theorem 1.2 .1 formulated below.

\subsection{Purity of dimension for functors associated with Koszul ma- trix factorizations}

Let $W\left(x_{1}, \ldots, x_{n}\right)$ be a quasihomogeneous polynomial of degree $d$, where $\operatorname{deg}\left(x_{i}\right)=d_{i}>0$, with an isolated singularity at the origin, and denote by $\operatorname{MF}_{\mathbb{G}_{m}}(W)$ the category of $\mathbb{G}_{m^{-}}$ equivariant matrix factorizations of $W$ (see Sec. 2.5 below). Here $\mathbb{G}_{m}$ acts on $\mathbb{A}^{n}$ by

$$
\lambda \cdot\left(x_{1}, \ldots, x_{n}\right)=\left(\lambda^{d_{1}} x_{1}, \ldots, \lambda^{d_{n}} x_{n}\right) .
$$

Let also $X$ be a smooth projective variety. We are going to prove a certain purity of dimension for the maps

$$
\mathcal{H}(W):=H H_{*}\left(\mathrm{MF}_{\mathbb{G}_{m}}(W)\right) \rightarrow H^{*}(X, \mathbb{C})
$$

induced by Fourier-Mukai functors

$$
\operatorname{DMF}_{\mathbb{G}_{m}}(W) \rightarrow D^{b}(X)
$$

of a special kind, where $\operatorname{DMF}_{\mathbb{G}_{m}}(W)$ is the derived category of $\mathbb{G}_{m}$-equivariant matrix factorizations of $W$.

Here is the precise setup. Assume that $A$ is a $\mathbb{G}_{m}$-equivariant vector bundle on $X$, where $\mathbb{G}_{m}$ acts trivially on $X$, equipped with a surjective $\mathbb{G}_{m}$-morphism of $\mathcal{O}_{X}$-modules

$$
z: A \rightarrow \mathcal{O}_{X}^{n},
$$

where $\mathbb{G}_{m}$ acts on $\mathcal{O}_{X}^{n}$ with the weights $\left(d_{1}, \ldots, d_{n}\right)$ Let $\operatorname{tot}(A)$ be the total space of this vector bundle, and let $p: \operatorname{tot}(A) \rightarrow X$ be the natural projection. Note that $z$ corresponds to a morphism

$$
Z: \operatorname{tot}(A) \rightarrow \mathbb{A}^{n},
$$


linear on the fibers of $p$ and $\mathbb{G}_{m}$-equivariant with respect to the action (1.4) on $\mathbb{A}^{n}$. Let $B$ be another $\mathbb{G}_{m}$-equivariant vector bundle on $X$ and suppose we have $\mathbb{G}_{m}$-morphisms of $\mathcal{O}_{X}$-modules

$$
\alpha: \oplus_{i=1}^{N} S^{i}(A) \rightarrow B^{\vee}\{d\}, \quad \beta: A \rightarrow B,
$$

where $S^{m}(\cdot)$ denotes the $m$ th symmetric power, and $\{d\}$ denotes the twist by the character $\lambda \mapsto \lambda^{d}$ of $\mathbb{G}_{m}$ We can view $\alpha$ and $\beta$ as $\mathbb{G}_{m}$-invariant sections of induced bundles on $\operatorname{tot}(A)$ :

$$
\alpha \in H^{0}\left(\operatorname{tot}(A), p^{*} B^{\vee}\{d\}\right), \quad \beta \in H^{0}\left(\operatorname{tot}(A), p^{*} B\right)
$$

The main assumption is that these sections satisfy

$$
\langle\alpha, \beta\rangle=-Z^{*} W
$$

and that the common vanishing locus of $\alpha$ and $\beta$ coincides with the zero section in $\operatorname{tot}(A)$. Then we have a $\mathbb{G}_{m}$-equivariant Koszul matrix factorization $\{\alpha, \beta\}$ of $-Z^{*} W$ on $\operatorname{tot}(A)$, supported at the zero section (see Sec. 2.5). We can use this matrix factorization and the diagram

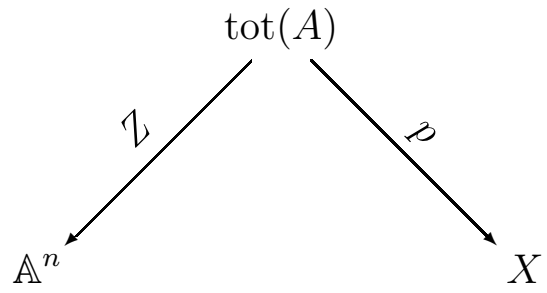

to define a Fourier-Mukai type functor

$$
\Phi: \mathrm{DMF}_{\mathbb{G}_{m}}\left(\mathbb{A}^{n}, W\right) \rightarrow D^{b}(X): E \mapsto p_{*}\left(Z^{*} E \otimes\{\alpha, \beta\}\right)
$$

(more precisely, this is the functor $\Phi_{P}$ for $P=\{\alpha, \beta\}$ defined in Sec. 2.6 below).

This functor has a natural realization on the dg-level which in particular allows to consider the induced map on the Hochschild homology

$$
\phi=\Phi_{*}: \mathcal{H}(W) \rightarrow H H_{*}(X) .
$$

The Hochschild-Kostant-Rosenberg isomorphism together with the Hodge theory give an identification $H H_{*}(X) \simeq H^{*}(X, \mathbb{C})$ (see Sec. 2.2).

Theorem 1.2.1. In the above situation assume in addition that the homogeneous polynomial $\widetilde{W}$ given by (1.3) still has an isolated singularity. Then for any $x \in \mathcal{H}(W)$ one has

$$
\operatorname{Td}(A)^{-1} \operatorname{Td}(B) \phi(x) \in H^{D}(X, \mathbb{C}) \subset H^{*}(X, \mathbb{C}),
$$

where $\operatorname{Td}(\cdot)$ denotes the Todd class, and

$$
D=2 \operatorname{rk} B-2 \operatorname{rk} A+n .
$$




\subsection{Outline of the proof}

The proof combines some ideas of noncommutative Hodge theory, the relation between graded matrix factorizations and derived categories of coherent sheaves on hypersurfaces (Orlov's equivalence), and a purity result from [33].

We start by rewriting the assertion using the left adjoint map to $\phi$ with respect to the canonical pairings on the Hochschild homology. Recall that for a smooth and proper dg category $\mathcal{C}$ the Hochschild homology is equipped with a canonical nondegenerate pairing $\langle\cdot, \cdot\rangle_{e}$, such that the maps on Hochschild homology induced by an adjoint pair of functors are adjoint with respect to the canonical pairings (see Sections 2.3 and 2.4 for details). Thus, the left adjoint map to $\phi$ is given by

$$
\psi=\Psi_{*}: H^{*}(X) \simeq H H_{*}(X) \rightarrow \mathcal{H}(W),
$$

where $\Psi: D^{b}(X) \rightarrow \operatorname{DMF}_{\mathbb{G}_{m}}(W)$ is the left adjoint functor to $\Phi$. Thus, we can rewrite the condition $\alpha \cdot \phi(\mathcal{H}(W)) \subset H^{D}(X)$, where $\alpha=\operatorname{Td}(A)^{-1} \operatorname{Td}(B)$, as

$$
\psi\left({ }^{\perp}\left(\alpha^{-1} H^{D}(X)\right)\right)=0 .
$$

Under the Hochschild-Kostant-Rosenberg isomorphism $H H_{*}(X) \simeq H^{*}(X)$ the canonical pairing takes the form

$$
\langle\cdot, \cdot\rangle_{D^{b}(X)}=\int_{X} \kappa(a) \cdot b \cdot \operatorname{Td}_{X},
$$

where $a, b \in H^{*}(X), \kappa$ is the linear operator on $H^{*}(X)$, such that $\kappa(c)=(-1)^{q} c$ for $c \in H^{p, q}$ (see Section 2.3). This implies that the left orthogonal to $\alpha^{-1} H^{D}(X) \subset H^{*}(X)$ with respect to the canonical pairing is

$$
{ }^{\perp}\left(\alpha^{-1} H^{D}(X)\right)=\bigoplus_{j \neq 2 \operatorname{dim} X-D} \kappa\left(\alpha \cdot \operatorname{Td}_{X}^{-1}\right) H^{j}(X),
$$

so we need to check that for each $y \in H^{j}(X)$, where $j \neq 2 \operatorname{dim} X-D$, one has

$$
\psi\left(\kappa\left(\alpha \cdot \operatorname{Td}_{X}^{-1}\right) \cdot y\right)=0 .
$$

Next, we recall that the Hochschild homology of the category of matrix factorizations has a canonical decomposition (see [36, Thm. 2.6.1])

$$
\mathcal{H}(W)=H H_{*}\left(\mathrm{MF}_{\mathbb{G}_{m}}(W)\right) \simeq \bigoplus_{\gamma \in \mu_{d}} H\left(W_{\gamma}\right)^{\mu_{d}},
$$

where $W_{\gamma}=\left.W\right|_{\left(\mathbb{A}^{n}\right)^{\gamma}}$, and $H(W)$ is given by (1.1). In fact, (1.8) is exactly the decomposition of $H H_{*}\left(\mathrm{MF}_{\mathbb{G}_{m}}(W)\right)$ into isotypical components with respect to the natural action of $\mathbb{Z} / d$ on it (see [36, Thm. 2.6.1(ii)]). Let $\Pi: \mathcal{H}(W) \rightarrow \mathcal{H}(W)$ denote the projector onto the summand $H(W)^{\mu_{d}}$, corresponding to $\gamma=1$. Using the characterization of this summand as $\mathbb{Z} / d$ invariants in $\mathcal{H}(W)$ we check that $\phi=\phi \Pi$ (see Lemma 2.6.3), and hence the image of $\psi$ is contained in $H(W)^{\mu_{d}} \subset \mathcal{H}(W)$. 
Now the idea is that $H(W)^{\mu_{d}}$ should be thought of as an analog of the primitive middle cohomology. Recall that if $X \subset \mathbb{P}^{N}$ is a smooth projective variety of dimension $n$ then the primitive part of the middle cohomology $P^{n}(X) \subset H^{n}(X)$ is defined as the kernel of the operator of multiplication with $c_{1}(H)$, where $H$ is the hyperplane class on $X$. The classical Hodge-Riemann relations imply (see Lemma 2.3.1) that for a nonzero class $a \in P_{H}^{n}(X)$ one has

$$
(-1)^{\frac{n(n+1)}{2}}\langle a, \bar{a}\rangle_{D^{b}(X)}>0,
$$

where $a \mapsto \bar{a}$ is the complex conjugation associated with the real structure on $H^{*}(X)$.

The key step of the proof is establishing an analog of this property for the canonical pairing on $H(W)^{\mu_{d}} \subset \mathcal{H}(W)$. One missing piece of structure that we need for this is a real structure on $\mathcal{H}(W)$. In fact, in general one expects to have a natural rational lattice in the Hochschild homology of any smooth proper dg-category (see [19], [4]). In the case of an admissible subcategory $\mathcal{C}$ of $D^{b}(Y)$ such a rational lattice can be constructed easily using the realization of its Hochschild homology as an image of a rational projector on $H^{*}(Y)$ (see Section 3.1). Furthermore, the obtained rational lattices are compatible with the maps on Hochschild homology induced by functors of Fourier-Mukai type.

Using the natural embedding $H(W)^{\mu_{d}} \subset H(\widetilde{W})^{\mu_{d}}$, where $W$ is given by (1.3) we reduce the situation to the homogeneous case (where $\operatorname{deg}\left(x_{i}\right)=1$ ). For homogeneous $W$, we apply Orlov's result, connecting the category of matrix factorizations $\operatorname{DMF}_{\mathbb{G}_{m}}(W)$ with the derived category of the corresponding projective hypersurface $X=(W=0)$, to realize $\operatorname{DMF}_{\mathbb{G}_{m}}(W)$ as such an admissible subcategory (see Lemma 3.2.1). Then using explicit descriptions of the canonical pairing $\langle\cdot, \cdot\rangle_{W}$ on $\mathcal{H}(W)$ and of the Chern characters of matrix factorizations in [34, we prove the following property of the subspace $H(W)^{\mu_{d}} \subset \mathcal{H}(W)$, which makes it an analogue of the primitive cohomology: $H(W)^{\mu_{d}}$ is orthogonal to the Chern characters of the matrix factorizations $k(m)^{\text {st }}$, for $m \in \mathbb{Z}$. Here $k(m)^{\text {st }}$ is the stabilization of the trivial module $k$, with the grading shifted by $m$. This is used in proving the analog of the Hodge-Riemann relations for matrix factorizations (see Proposition 3.2.4), which states that for a nonzero class $x \in H(W)^{\mu_{d}} \cap \mathcal{H}_{j}(W)$ one has $\langle x, \bar{x}\rangle_{W} \neq 0$ (and in fact, $\langle x, \bar{x}\rangle_{W}$ is a positive multiple of a certain power of $i$ ). Roughly speaking, this is proved by reducing to the classical Hodge-Riemann relations for cohomology classes on the projective hypersurface $X$, using Orlov's theorem relating the categories $\operatorname{DMF}_{\mathbb{G}_{m}}(W)$ and $D^{b}(X)$. For example, in the case $d=n$ Orlov's theorem states an equivalence of these categories, and we check that $H(W)^{\mu_{d}}$ corresponds precisely to the primitive cohomology of $X$ under the corresponding isomorphism between the Hochschild homology (see Remark 3.2.5).

Since the image of $\psi$ is contained in $H(W)^{\mu_{d}}$, the above Hodge-Riemann relations show that the vanishing (1.7) is equivalent to the vanishing

$$
\left\langle\overline{\psi\left(y^{\prime}\right)}, \psi\left(y^{\prime}\right)\right\rangle_{W}=0,
$$

where $y^{\prime}=\kappa\left(\alpha \cdot \operatorname{Td}_{X}^{-1}\right) \cdot y$ and $y \in H^{p, q}$ with $p+q \neq 2 \operatorname{dim} X-D$. Using the adjointness of $\phi$ and $\psi$ we rewrite this as a certain purity property for the composition $\phi \psi=(\Phi \circ \Psi)_{*}$.

Finally, a computation with the Fourier-Mukai kernels (see Sections 2.6 and 4.1) shows that the kernel $K$ on $X \times X$ defining the functor $\Phi \circ \Psi: D^{b}(X) \rightarrow D^{b}(X)$ is given by the push-forward of a Koszul matrix factorization of zero on some vector bundle over $X \times X$, 
supported along the zero section. Applying the results of [33] (see Prop. 4.1.1) we obtain that the appropriate twist of the class $\operatorname{ch}(K)$ is pure of certain dimension (see (4.1)). This gives the required purity property for $\phi \psi$ and so finishes the proof.

We conjecture that a statement similar to Theorem 1.2.1 holds for any quasihomogeneous $W$ with an isolated singularity. One could try to mimic our proof in the homogeneous case. However, at present several technical ingredients are lacking. For example, in this case the analog of the projective hypersurface is a DM-stack, so we need to identify the canonical bilinear form on the Hochschild homology of a smooth proper DM-stack in terms of the Hochschild-Kostant-Rosenberg isomorphism. Another problem is matching the effect of Orlov's equivalence on the Hochschild homology with the ad hoc isomorphism constructed in [11.

The paper is organized as follows. In Section 2 we build the background and prove some technical statements. The most important bits are Section 2.6, where we establish an adjunction result for Fourier-Mukai type functors involving matrix factorizations, and Section 2.7] containing some calculations with Orlov's equivalence. Then in Section 3 we discuss rational lattices and prove the analog of the Hodge-Riemann bilinear relations for the Hochschild homology of the category of matrix factorizations of a homogeneous polynomial with an isolated singularity (or a quasihomogeneous polynomial as in Theorem 1.2.1, see Proposition 3.2.4). Finally, in Section 4 we recall the purity result from [33] and show how Theorem 1.2.1 is deduced from it. We then deduce Theorem 1.1.1 in Section 4.3. In the Appendix we prove a technical result involving Grothendieck duality and matrix factorizations, which is needed in Section 2.6.

Conventions. We work with schemes and dg-categories over a field $k$. Starting from Section 3 we assume that $k=\mathbb{C}$. For a smooth projective variety $X$ we denote by $D^{b}(X)$ the bounded derived category of coherent sheaves on $X$, which we equip with one of the standard dgenhancements. We denote by $\operatorname{Per}(X) \subset D^{b}(X)$ the full subcategory of perfect complexes. For an algebraic group $G$ acting on $X$ we denote by $\operatorname{Per}_{G}(X)$ the category of $G$-equivariant perfect complexes. For a morphism of schemes $f$ we denote by $f^{*}$ and $f_{*}$ the corresponding derived functors of pull-back and push-forward. For an additive category $\mathcal{C}$ we denote by $\overline{\mathcal{C}}$ its Caroubian completion. By $\operatorname{ch}(\cdot)$ and $\operatorname{Td}(\cdot)$ we denote the characteristic classes of algebraic vector bundles constructed using the Atiyah class, as in [1] (their components differ from the corresponding topological characteristic classes by factors of $(-2 \pi i))$.

Acknowledgments. I would like to thank Alessandro Chiodo and Dimitri Zvonkine for organizing the workshop "Mirror symmetry and spin curves" which prompted me to write this paper. I am grateful to Nick Rosenblyum for a useful discussion on adjoint functors. I am also grateful to the Mathematical Sciences Research Institute at Berkeley, where some of this work was done, for the hospitality and excellent working conditions. Finally, I thank the anonimous referee for useful suggestions. This research is supported in part by the NSF grant DMS-1400390. 


\section{Preliminaries}

\subsection{DG-categories and dg-functors}

Let $k$ be a field. For a dg-category $\mathcal{C}$ over $k$ we denote by $D(\mathcal{C})$ the derived category of right $\mathcal{C}$-modules and by $\operatorname{Per}(\mathcal{C}) \subset D(\mathcal{C})$ the subcategory of perfect modules. We denote by $\operatorname{Per}_{d g}(\mathcal{C})$ the natural dg-enhancement of $\operatorname{Per}(\mathcal{C})$ (defined using cofibrant right $\mathcal{C}$-modules, see [41, Sec. 7]).

Throughout this paper we consider only $\mathcal{C}$ such that $\operatorname{Per}_{d g}(\mathcal{C})$ is saturated (see [42, Sec. $2.2])$.

For an object $K \in \operatorname{Per}\left(\mathcal{C}^{o p} \otimes \mathcal{D}\right)$ we have a dg-functor of tensoring with $K$,

$$
\Phi_{K}: \operatorname{Per}_{d g}(\mathcal{C}) \rightarrow \operatorname{Per}_{d g}(\mathcal{D}): M \mapsto M \otimes_{\mathcal{C}}^{\mathbb{L}} K
$$

It is known that in this way we get a bijection between isomorphism classes in $\operatorname{Per}\left(\mathcal{C}^{o p} \otimes \mathcal{D}\right)$ and morphisms between $\operatorname{Per}_{d g}(\mathcal{C})$ and $\operatorname{Per}_{d g}(\mathcal{D})$ in the localized category Hqe of dg-categories, obtained by inverting quasi-equivalences (see [41]). To a usual dg-functor $\Phi: \mathcal{C} \rightarrow \mathcal{D}$ we associate a bimodule, i.e., an object in $D\left(\complement^{o p} \otimes \mathcal{D}\right)$,

$$
(C, D) \mapsto \operatorname{Hom}_{\mathcal{D}}(D, \Phi(C))
$$

which is perfect under our assumptions on $\mathcal{C}$ and $\mathcal{D}$. The corresponding tensor functor $\operatorname{Per}_{d g}(\mathcal{C}) \rightarrow \operatorname{Per}_{d g}(\mathcal{D})$ is an extension of $\Phi$.

In the remainder of the paper we often switch between $K$ and $\Phi_{K}$, and sometimes, denote them by the same letter. We denote by $\Delta_{\mathcal{C}} \in \operatorname{Per}\left(\mathcal{C}^{o p} \otimes \mathcal{C}\right)$ the diagonal bimodule corresponding to the identity functor. We also have the corresponding dg-functor

$$
\operatorname{Tr}_{\mathcal{C}}^{d g}: \operatorname{Per}_{d g}\left(\mathfrak{C}^{o p} \otimes \mathcal{C}\right) \rightarrow \operatorname{Per}_{d g}(k)
$$

mapping $A^{\vee} \otimes B$ to $\operatorname{Hom}_{\mathfrak{C}}(A, B)$.

For a pair of kernels $K_{1} \in \operatorname{Per}\left(\mathcal{C}_{1}^{o p} \otimes \mathcal{D}_{1}\right), K_{2} \in \operatorname{Per}\left(\mathfrak{C}_{2}^{o p} \otimes \mathcal{D}_{2}\right)$ we have the induced functor

$$
K_{1} \square K_{2}: \operatorname{Per}_{d g}\left(\mathcal{C}_{1} \otimes \mathcal{C}_{2}\right) \rightarrow \operatorname{Per}_{d g}\left(\mathcal{D}_{1} \otimes \mathcal{D}_{2}\right),
$$

given by the external tensor product of the kernels $K_{1}$ and $K_{2}$. Recall that there is a natural equivalence

$$
\operatorname{Per}(\mathcal{C})^{o p} \stackrel{\sim}{\longrightarrow} \operatorname{Per}\left(\mathrm{C}^{o p}\right): M \mapsto M^{\vee}
$$

(see e.g., [39, (3.6)]). Using this equivalence, for a kernel $F \in \operatorname{Per}\left(\mathcal{C}^{o p} \otimes \mathcal{D}\right)$ we can define $F^{o p} \in \operatorname{Per}\left(\mathcal{C} \otimes \mathcal{D}^{o p}\right)$ as the kernel corresponding to the functor

$$
\operatorname{Per}\left(\mathcal{C}^{o p}\right) \stackrel{\sim}{\longrightarrow} \operatorname{Per}(\mathcal{C})^{o p} \stackrel{F}{\longrightarrow} \operatorname{Per}(\mathcal{D})^{o p} \stackrel{\sim}{\longrightarrow} \operatorname{Per}\left(\mathcal{D}^{o p}\right) .
$$

Definition 2.1.1. Let $F \in \operatorname{Per}\left(\mathfrak{C}^{o p} \otimes \mathcal{D}\right), G \in \operatorname{Per}\left(\mathcal{D}^{o p} \otimes \mathfrak{C}\right)$. We say that $(F, G)$ is an adjoint pair, or that $F$ is left adjoint to $G$ if a morphism

$$
\varphi: \Delta_{\mathcal{C}} \rightarrow G \circ F
$$


is given in $D\left(\mathfrak{C}^{o p} \otimes \mathfrak{C}\right)$, such that for any $C \in \operatorname{Per}(\mathcal{C})$ and $D \in \operatorname{Per}(\mathcal{D})$ the induced morphism

$$
\operatorname{Hom}_{\mathcal{D}}(F(C), D) \stackrel{G}{\longrightarrow} \operatorname{Hom}_{\mathcal{C}}(G F(C), G(D)) \stackrel{? \circ \varphi}{\longrightarrow} \operatorname{Hom}_{\mathcal{C}}(C, G(D))
$$

is a quasi-isomorphism.

Note that if $(F, G)$ is an adjoint pair then the map (2.2) can be extended to a similar map of dg-functors $\operatorname{Per}_{d g}\left(\mathrm{C}^{o p} \otimes \mathcal{D}\right) \rightarrow \operatorname{Per}_{d g}(k)$,

$$
\operatorname{Tr}_{\mathcal{D}} \circ\left(F^{o p} \square \Delta_{\mathcal{D}}\right) \rightarrow \operatorname{Tr}_{\mathcal{C}} \circ\left(\operatorname{Id}_{\mathcal{C}} \square G\right)
$$

induced by a quasi-isomorphism of kernels in $\operatorname{Per}\left(\mathcal{C} \otimes \mathcal{D}^{o p}\right)$. Indeed, the fact that (2.3) is a quasi-isomorphism of kernels is equivalent to the assertion that it becomes a quasiisomorphism of complexes when applied to $C^{\vee} \otimes D \in \mathcal{C}^{o p} \otimes \mathcal{D}$, which corresponds to (2.2) being a quasi-isomorphism.

Recall that a $\mathbb{Z} / 2$-dg-category is a dg-category in which all Hom-complexes are 2-periodic. Equivalently, we can replace the 2-periodic complexes by the corresponding $\mathbb{Z} / 2$-graded complexes. The theory of $\mathbb{Z} / 2$-dg-categories is parallel to the theory of dg-categories (see [14, Sec. 4.1]). With each dg-category $\mathcal{C}$ we can associate its $\mathbb{Z} / 2$-folding $\mathcal{C}^{(2)}$, which is a $\mathbb{Z} / 2$-dg-category such that

$$
\operatorname{Hom}_{\mathcal{C}^{(2)}}^{0}(A, B)=\bigoplus_{i \in \mathbb{Z}} \operatorname{Hom}_{\mathcal{C}}^{2 i}(A, B), \operatorname{Hom}_{\mathcal{C}^{(2)}}^{1}(A, B)=\bigoplus_{i \in \mathbb{Z}} \operatorname{Hom}_{\mathcal{C}}^{2 i+1}(A, B) .
$$

One can think of this operation as the tensor product with the algebra $k\left[u, u^{-1}\right]$, where $\operatorname{deg}(u)=2$. It is easy to see that the Hochschild homology functor commutes with passing to the $\mathbb{Z} / 2$-folding, i.e., the Hochschild homology of $\mathrm{C}^{(2)}$ as a $\mathbb{Z} / 2$-dg-category is the $\mathbb{Z} / 2$ folding of the complex $H H_{*}(\mathcal{C})$, and these identifications are compatible with the maps induced by functors. In particular, taking the Chern character and the canonical pairing on the Hochschild homology discussed below are also compatible with this operation.

\subsection{Chern character in Hochschild homology versus topological Chern character}

Recall that for any dg-category $\mathcal{C}$ over $k$ and an object $E$ of $\mathcal{C}$ one has the Chern character $\operatorname{ch}(E) \in H H_{0}(\mathcal{C})$ defined by the functoriality of the Hochschild homology (see e.g., [39], [34]).

In the case when $\mathrm{e}=D^{b}(X)$, the dg-version of the bounded derived category of coherent sheaves on a smooth projective variety $X$ over $\mathbb{C}$, we have the Hochschild-Kostant-Rosenberg isomorphism

$$
H H_{*}\left(D^{b}(X)\right)=H H_{*}(X) \simeq \oplus_{p, q} H^{q}\left(X, \Omega^{p}\right)
$$

so that $H_{i}$ corresponds to the sum of terms with $p-q=i$. Hence, by Hodge theory, we can identify the Hochschild homology $H H_{*}(X)$ with $H^{*}(X, \mathbb{C})$.

As was shown in [6], under this identification, the abstract Chern character with values in $H H_{*}(X)$ is essentially the same as the topological Chern character with values in $H^{*}(X, \mathbb{C})$ (for $k=\mathbb{C}$ ). Here is a more precise statement. 
Proposition 2.2.1. Let $k=\mathbb{C}$. For $E \in D^{b}(X)$ let $\operatorname{ch}^{\text {top }}(E) \in H^{*}(X, \mathbb{C})$ denote the usual topological Chern character. Then one has

$$
\operatorname{ch}_{k}(E)=(-2 \pi i)^{k} \operatorname{ch}_{k}^{t o p}(E) .
$$

Proof. Caldararu computes $\operatorname{ch}(E)$ in terms of traces of powers of the Atiyah class $\operatorname{At}(E) \in$ $\operatorname{Ext}^{1}\left(E, E \otimes \Omega^{1}\right)$ (see the proof of [6, Thm. 4.5]). Thus, the assertion follows from the comparison with the topological Chern character in [1, Sec. 5].1]

In what follows we always use the notation $\operatorname{ch}(\cdot)$ for the abstract Chern character defined using Hochschild functoriality (or in terms of the Atiyah class, in the case of $D^{b}(X)$ ). We denote by $\operatorname{Td}(\cdot)$ the Todd class defined by the standard formulas in terms of the components of $\operatorname{ch}(\cdot)$ (we only use it for $D^{b}(X)$ ).

\subsection{Canonical pairing on the Hochschild homology}

Applying the functoriality of the Hochschild homology to the functor (2.1) and using the Künneth isomorphism for Hochschild homology we obtain a canonical pairing

$$
\langle\cdot, \cdot\rangle_{\complement}: H H_{*}\left(\complement^{o p}\right) \otimes H H_{*}(\mathfrak{C}) \rightarrow k
$$

(cf. [39, Sec. 1.2]).

Note that there is a canonical isomorphism $H H_{*}\left(\mathrm{C}^{o p}\right) \simeq H H_{*}(\mathrm{C})$, so we can think of the canonical pairing as a pairing on $H H_{*}(\mathcal{C})$. However, one should be careful that in the case when the category $\mathfrak{C}$ is equipped with the duality $\mathfrak{C}^{o p} \simeq \mathcal{C}$ the induced identification of $H H_{*}\left(\mathcal{C}^{o p}\right) \simeq H H_{*}(\mathcal{C})$ may be different from the canonical one. For example this is the case for $\mathcal{C}=D^{b}(X)$, where it is customary to use the duality on sheaves to identify $\mathcal{C}$ with $\mathcal{C}^{o p}$. This is related to the involution $a \mapsto \kappa(a)$ on the Hochschild homology of $X$ arising below (cf. [32, Rem. 2.15]).

In the case of $\mathcal{C}=D^{b}(X)$ (where $X$ is a smooth projective variety) the canonical pairing on $H H_{*}\left(D^{b}(X)\right)$ is given in terms of the HKR-isomorphism (2.4) by the formula

$$
\langle a, b\rangle_{D^{b}(X)}=(\kappa(a), b)_{X},
$$

where

$$
\begin{gathered}
(a, b)_{X}:=\int_{X} a \cdot b \cdot \operatorname{Td}_{X}, \\
\kappa(c)=(-1)^{q} c \text { for } c \in H^{p, q}=H^{q}\left(X, \Omega^{p}\right)
\end{gathered}
$$

(see [37, Eq. (8)]; note that Ramadoss in [37] works with the Mukai pairing which coincides with the canonical pairing (2.5) for $\mathcal{C}=D^{b}(X)$, e.g., by [32, Prop. 2.14]). Here $\int_{X}$ is defined as the projection

$$
\int_{X}: \oplus_{p, q} H^{q}\left(X, \Omega^{p}\right) \rightarrow H^{n}\left(X, \Omega_{X}^{n}\right) \simeq k,
$$

\footnotetext{
${ }^{1}$ The factor $(-2 \pi i)^{k}$ appears due to the standard normalization of $\operatorname{ch}^{\text {top }}(E)$, so that it takes values in $H^{*}(X, \mathbb{Z})$. Caldararu uses a different normalization, so he does not have this factor.
} 
where $n=\operatorname{dim} X$. In the case $k=\mathbb{C}$ it is related to the topological operation of integration over the fundamental cycle of $X$ by the formula

$$
\int_{X} \omega=\frac{1}{(2 \pi i)^{n}} \int_{[X]}^{t o p} \omega
$$

(see e.g., [13]).

The classical Hodge-Riemann bilinear relations (see e.g., [43, Ch. V]) imply the following property of the canonical pairing $\langle\cdot, \cdot\rangle_{D^{b}(X)}$.

Lemma 2.3.1. Let $H \in \operatorname{Pic}(X)$ be an ample class, and let $P_{H}^{n}(X) \subset H^{n}(X)$ be the corresponding primitive part of the middle cohomology consisting of the classes a such that $a \cdot c_{1}(H)=0$. Then for a nonzero class $a \in P_{H}^{n}(X)$ one has

$$
(-1)^{\frac{n(n+1)}{2}}\langle a, \bar{a}\rangle_{D^{b}(X)}>0 .
$$

Proof. By (2.6), we have

$$
\langle a, \bar{a}\rangle_{D^{b}(X)}=(-1)^{q} \int_{X} a \cdot \bar{a} \cdot \operatorname{Td}_{X}=\frac{(-1)^{q}}{(2 \pi i)^{n}} \int_{[X]}^{t o p} a \cdot \bar{a} \cdot \operatorname{Td}_{X},
$$

where $a \in H^{p, q}, p+q=n$. Since in our case $a \cdot \bar{a} \in H^{n, n}(X)$, we can delete $\mathrm{Td}_{X}$, and the result follows from the Hodge-Riemann bilinear relations stating that

$$
(-1)^{\frac{n(n-1)}{2}} i^{p-q} \int_{[X]}^{t o p} a \cdot \bar{a}>0 .
$$

\subsection{Maps on Hochschild homology induced by Fourier-Mukai func- tors}

We need the fact that adjoint functors induce adjoint operators on Hochschild homology with respect to the canonical pairings (2.5) (cf. [8, Thm. 8] in the case of derived categories of sheaves).

Lemma 2.4.1. If $\left(F \in \operatorname{Per}\left(\mathcal{C}^{o p} \otimes \mathcal{D}\right), G \in \operatorname{Per}\left(\mathcal{D}^{o p} \otimes \mathcal{C}\right)\right)$ is an adjoint pair of kernels then

$$
\left\langle F_{*}(x), y\right\rangle_{\mathcal{D}}=\left\langle x, G_{*}(y)\right\rangle_{\mathcal{e}},
$$

where $x \in H H_{*}(\mathcal{C}), y \in H H_{*}(\mathcal{D})$, and $F_{*}: H H_{*}(\mathcal{C}) \rightarrow H H_{*}(\mathcal{D}), G_{*}: H H_{*}(\mathcal{D}) \rightarrow H H_{*}(\mathcal{C})$ are the induced maps on the Hochschild homology.

Proof. The required equality is immediately obtained from (2.3) by passing to the induced maps on Hochschild homology. We have to use the fact that the map

$$
F_{*}^{o p}: H H_{*}\left(\bigodot^{o p}\right) \rightarrow H H_{*}\left(\mathcal{D}^{o p}\right)
$$


coincides with $F_{*}$ under the natural identifications $H H_{*}(\mathcal{C}) \simeq H H_{*}\left(\mathrm{C}^{o p}\right), H H_{*}(\mathcal{D}) \simeq H H_{*}\left(\mathcal{D}^{o p}\right)$. The simplest way to check this is to use the definition of the maps $F_{*}$ via the Hochschild chain complexes.

We will use the following formula for the maps induced on the Hochschild homology by Fourier-Mukai type functors, in terms of the Chern character of the kernel and the pairing (2.7).

Lemma 2.4.2. For the Fourier-Mukai functor $F: D^{b}(X) \rightarrow D^{b}(Y)$ associated with a kernel $K \in D^{b}(X \times Y)$ the induced map on the Hochschild homology gets identified via the HKRisomorphisms with

$$
F_{*}: H^{*}(X, \mathbb{C}) \rightarrow H^{*}(Y, \mathbb{C}): a \mapsto \operatorname{tr}_{12}(a \otimes \operatorname{ch}(K)),
$$

where

$$
\operatorname{tr}_{12}: H^{*}(X) \otimes H^{*}(X) \otimes H^{*}(Y) \rightarrow H^{*}(Y): a \otimes b \otimes c \mapsto(a, b)_{X} c
$$

Proof. This is equivalent to [26, Thm. 1.2].

\subsection{Matrix factorizations}

In this section we recall some basic results about matrix factorizations and also prove several technical statements that will be needed later in working with Fourier-Mukai transforms involving matrix factorizations. The key result that allows to deduce many results for matrix factorizations from the classical results about coherent sheaves is the equivalence with the singularity category of the hypersurface $W=0$ (see (2.9) below).

Let $X$ be a $\mathbb{G}_{m}$-scheme, i.e., a scheme with a $\mathbb{G}_{m}$-action. Throughout this paper we make an assumption that our $\mathbb{G}_{m}$-schemes admit a $\mathbb{G}_{m}$-equivariant ample line bundle. This implies that they admit a $\mathbb{G}_{m}$-invariant open affine cover and have a resolution property, i.e., every $\mathbb{G}_{m}$-equivariant coherent sheaf on $X$ admits a surjection from a $\mathbb{G}_{m}$-equivariant vector bundle (see [40]).

For a $\mathbb{G}_{m}$-equivariant quasicoherent sheaf $\mathcal{F}$ on $X$ we denote by $\mathcal{F}\{i\}$ the same sheaf with the $\mathbb{G}_{m}$-action twisted by the character $\lambda \mapsto \lambda^{i}$ of $\mathbb{G}_{m}$.

Let $W$ be a function on $X$ of weight $d>0$ with respect to the $\mathbb{G}_{m}$-action, i.e., $W \in$ $H^{0}\left(X, \mathcal{O}_{X}\{d\}\right)^{\mathbb{G}_{m}}$. A $\mathbb{G}_{m}$-equivariant matrix factorization of $W$ is a $\mathbb{Z} / 2$-graded vector bundle $E=E_{0} \oplus E_{1}$ on $X$ together with maps

$$
\delta_{1}: E_{1} \rightarrow E_{0}, \quad \delta_{0}: E_{0} \rightarrow E_{1}\{d\}
$$

such that $\delta_{0} \delta_{1}=W \cdot$ id and $\delta_{1} \delta_{0}=W \cdot$ id. For a pair of $\mathbb{G}_{m}$-equivariant matrix factorizations of $W, E$ and $F$ we consider the complex of $\mathbb{G}_{m}$-equivariant sheaves $\underline{\operatorname{Hom}}(E, F)$ given by

$$
\begin{gathered}
\underline{\operatorname{Hom}}^{2 n}(E, F)=\underline{\operatorname{Hom}}\left(E_{0}, F_{0}\{d n\}\right) \oplus \underline{\operatorname{Hom}}\left(E_{1}, F_{1}\{d n\}\right), \\
\underline{\operatorname{Hom}}^{2 n+1}(E, F)=\underline{\operatorname{Hom}}\left(E_{0}, F_{1}\{d(n+1)\}\right) \oplus \underline{\operatorname{Hom}}\left(E_{1}, F_{0}\{d n\}\right),
\end{gathered}
$$


with the differential $f \mapsto \delta_{F} \circ f-(-1)^{|f|} f \circ \delta_{E}$. Let

$$
R \Gamma(X, \cdot): \operatorname{Com}\left(\mathrm{Qcoh}_{\mathbb{G}_{m}}(X)\right) \rightarrow \operatorname{Com}\left(k\left[\mathbb{G}_{m}\right]\right)
$$

be a multiplicative dg-model of the push-forward to the point, given e.g., by the Cech complex with respect to a $\mathbb{G}_{m}$-invariant open affine cover (as in [38, Sec. 2]). We define the dg-category $\mathrm{MF}_{\mathbb{G}_{m}}(X, W)$ of $\mathbb{G}_{m}$-equivariant matrix factorizations of $W$ by setting

$$
\operatorname{Hom}(E, F)=R \Gamma(X, \underline{\operatorname{Hom}}(E, F))^{\mathbb{G}_{m}} .
$$

Passing to the 0th cohomology of the dg-category $\mathrm{MF}_{\mathbb{G}_{m}}(X, W)$ we get the derived category of matrix factorizations $\operatorname{DMF}_{\mathbb{G}_{m}}(X, W)$.

Assume that $X$ is smooth. Recall that by our assumption $X$ admits a $\mathbb{G}_{m}$-equivariant ample line bundle, hence it has a $\mathbb{G}_{m}$-resolution property. Assume also that $W$ is not a zero divisor. Then the functor associating with a matrix factorization $E$ the cokernel of $\delta_{1}: E_{1} \rightarrow E_{0}$ extends to an equivalence

$$
\mathrm{DMF}_{\mathbb{G}_{m}}(X, W) \stackrel{\sim}{\longrightarrow} D_{\mathrm{Sg}, \mathbb{G}_{m}}\left(X_{0}\right),
$$

where $X_{0} \subset X$ is the hypersurface $W=0, D_{\mathrm{Sg}, \mathbb{G}_{m}}\left(X_{0}\right)$ is the singularity category, defined as the quotient of the bounded $\mathbb{G}_{m}$-equivariant derived category by the subcategory $\operatorname{Per}_{\mathbb{G}_{m}}\left(X_{0}\right)$ of perfect complexes. In this form the equivalence follows from [35, Thm. 3.14] but the construction and the main ideas go back to Orlov [28] (see also [29, Sec. 3], [30] and [15]).

Note that there is a different way to define the derived category of matrix factorizations as the absolute derived category $D^{\mathrm{abs}}\left(\mathrm{MF}_{\mathbb{G}_{m}}(X, W)\right)$, which is the quotient of the naive homotopy category by the convolutions of exact sequences of matrix factorizations (see [30], [15]). The equivalence of this definition with the one above (in the case of smooth $X$ ) follows from the equivalence

$$
D_{\mathbb{G}_{m}}^{\mathrm{abs}}(X, W) \simeq D_{\mathrm{Sg}, \mathbb{G}_{m}}\left(X_{0}\right)
$$

(which can be proved as in [30], [15]). In the case when $X$ is affine (and smooth) the derived category of matrix factorization on $X$ coincides with the naive homotopy category (see e.g., [29, Sec. 3], [35, Prop. 3.19]).

For technical reasons we often work with the Caroubian completion $\overline{\operatorname{DMF}}_{\mathbb{G}_{m}}(X, W)$ of the derived category of matrix factorizations. It can be realized as a full subcategory in the derived category of matrix factorizations of quasicoherent sheaves (see [35, Sec. 4], [15, Sec. 2.3]). In the case when $W$ is a homogeneous polynomial on $\mathbb{A}^{n}$ with an isolated singularity the relation of $\mathrm{DMF}_{\mathbb{G}_{m}}\left(\mathbb{A}^{n}, W\right)$ with the derived category $D^{b}(Y)$ on the corresponding projective hypersurface $Y$ (see Sec. 2.7) implies that $\mathrm{DMF}_{\mathbb{G}_{m}}\left(\mathbb{A}^{n}, W\right)$ is in fact Caroubian closed.

We have a natural duality equivalence

$$
\mathbb{D}: \mathrm{MF}_{\mathbb{G}_{m}}(X, W)^{o p} \rightarrow \mathrm{MF}_{\mathbb{G}_{m}}(X,-W),
$$

where $\mathbb{D}(E)_{0}=E_{0}^{\vee}, \mathbb{D}(E)_{1}=E_{1}^{\vee}\{-d\}$ with the induced differential (see [34, Eq. (2.13)]). Often, we will simply write $E^{\vee}$ instead of $\mathbb{D}(E)$. Similarly to the $\mathbb{Z} / 2$-graded case (see [25, Lem. 3.9]) this duality can be interpreted in terms of the singularity category. 
Lemma 2.5.1. Assume that $X$ is smooth and $W$ is not a zero divisor. Under the equivalences of $\mathrm{DMF}_{\mathbb{G}_{m}}(X, W)$ and $\mathrm{DMF}_{\mathbb{G}_{m}}(X,-W)$ with $D_{\mathrm{Sg}, \mathbb{G}_{m}}^{b}\left(X_{0}\right)$, the duality $\mathbb{D}$ corresponds to the duality $F \mapsto R \underline{\operatorname{Hom}}\left(F, \mathcal{O}_{X_{0}}[-1]\right)$.

Proof. Recall that for a matrix factorization $E$ the corresponding object of the singularity category is represented by the coherent sheaf $F$ on $X_{0}$ fitting into the exact sequence

$$
0 \rightarrow E_{1} \rightarrow E_{0} \rightarrow i_{*} F \rightarrow 0
$$

where $i: X_{0} \rightarrow X$ is the embedding. By duality, we have an exact triangle

$$
E_{0}^{\vee} \rightarrow E_{1}^{\vee} \rightarrow R \underline{\operatorname{Hom}}\left(i_{*} F, \mathcal{O}_{X}\right)[1] \rightarrow \ldots
$$

By Grothendieck duality,

$$
R \underline{\operatorname{Hom}}\left(i_{*} F, \mathcal{O}_{X}\right)[1] \simeq i_{*} R \underline{\operatorname{Hom}}\left(F, i^{!} \mathcal{O}_{X}\right)[1] \simeq i_{*} R \underline{\operatorname{Hom}}\left(F, \mathcal{O}_{X_{0}}\right)
$$

Thus, $G=R \underline{\operatorname{Hom}}\left(F, \mathcal{O}_{X_{0}}\right)$ is a sheaf on $X_{0}$, and we have an exact sequence

$$
0 \rightarrow E_{0}^{\vee} \rightarrow E_{1}^{\vee} \rightarrow i_{*} G \rightarrow 0
$$

On the other hand, the object of the singularity category associated with $\mathbb{D}(E)$ is the coherent sheaf $F^{\prime}$ on $X_{0}$ from the exact sequence

$$
0 \rightarrow E_{1}^{\vee}\{-d\} \rightarrow E_{0}^{\vee} \rightarrow i_{*} F^{\prime} \rightarrow 0 .
$$

Hence, we have an exact sequence on $X_{0}$

$$
0 \rightarrow F^{\prime} \rightarrow i^{*} E_{1}^{\vee} \rightarrow G \rightarrow 0
$$

which shows that $F^{\prime} \simeq G[-1]$ in the singularity category.

For a pair of potentials $W, W^{\prime}$ on $X$, both of weight $d>0$, we define the tensor product functor

$$
\otimes: \mathrm{MF}_{\mathbb{G}_{m}}(X, W) \times \mathrm{MF}_{\mathbb{G}_{m}}\left(X, W^{\prime}\right) \rightarrow \mathrm{MF}_{\mathbb{G}_{m}}\left(X, W+W^{\prime}\right)
$$

by setting

$$
(E \otimes F)_{0}=E_{0} \otimes F_{0} \oplus E_{1} \otimes F_{1}\{d\} \quad \text { and }(E \otimes F)_{0}=E_{0} \otimes F_{1} \oplus E_{1} \otimes F_{0}
$$

with the differential $\delta_{E} \otimes \mathrm{id}_{F}+\mathrm{id}_{E} \otimes \delta_{F}$.

For $F \in \mathrm{MF}_{\mathbb{G}_{m}}(X, W)$ we can consider the infinite complex of $\mathbb{G}_{m}$-equivariant sheaves on $X_{0}=W^{-1}(0)$,

$$
\operatorname{com}(F):\left.\left.\left.\left.\ldots \rightarrow E_{0}\{-d\}\right|_{X_{0}} \rightarrow E_{1}\right|_{X_{0}} \rightarrow E_{0}\right|_{X_{0}} \rightarrow E_{1}\{d\}\right|_{X_{0}} \rightarrow \ldots
$$

with $\left.E_{0}\right|_{X_{0}}$ placed in degree 0 . Note that if $W=0$ then this is a complex on $X=X_{0}$. The following relation between Hom's, duality and tensor product is straightforward to check (cf. [36, Lem. 1.1.6]). 
Lemma 2.5.2. For $E, F \in \mathrm{MF}_{\mathbb{G}_{m}}(X, W)$ one has an isomorphism of $\mathbb{G}_{m}$-equivariant complexes on $X$,

$$
\underline{\operatorname{Hom}}(E, F) \simeq \operatorname{com}\left(E^{\vee} \otimes F\right)
$$

Hence,

$$
\operatorname{Hom}(E, F) \simeq R \Gamma\left(X, \operatorname{com}\left(E^{\vee} \otimes F\right)\right)^{\mathbb{G}_{m}}
$$

For a $\mathbb{G}_{m}$-scheme $X$ and for some $d>0$ we can consider the category $\operatorname{MF}_{\mathbb{G}_{m}, d}(X, 0)$ of matrix factorizations of 0 on $X$, where 0 is viewed as a function of weight $d$. In this situation we define the functor

$$
\mathrm{mf}: \operatorname{Per}_{\mathbb{G}_{m}}(X) \rightarrow \mathrm{DMF}_{\mathbb{G}_{m}, d}(X, 0): \operatorname{mf}\left(C^{\bullet}\right)_{0}=\bigoplus_{n} C^{2 n}\{-n d\}, \operatorname{mf}\left(C^{\bullet}\right)_{1}=\bigoplus_{n} C^{2 n-1}\{-n d\}
$$

(note that since we have a resolution property for $\mathbb{G}_{m}$-equivariant sheaves on $X$, an object of $\operatorname{Per}_{\mathbb{G}_{m}}(X)$ can be represented globally by a bounded complex of $\mathbb{G}_{m}$-equivariant vector bundles, see [35, Lem. 3.5]). If $W$ is a function on $X$ of weight $d$ then we also get the tensor product operation

$$
\otimes: \operatorname{Per}_{\mathbb{G}_{m}}(X) \times \mathrm{DMF}_{\mathbb{G}_{m}}(X, W) \rightarrow \mathrm{DMF}_{\mathbb{G}_{m}}(X, W), \quad F \otimes E:=\operatorname{mf}(F) \otimes E
$$

Then one has a natural isomorphism of complexes on $X_{0}$,

$$
\left.\operatorname{com}(F \otimes E) \simeq F\right|_{X_{0}} \otimes \operatorname{com}(E)
$$

(see [36, Lem. 1.1.5]). This easily implies (assuming $W$ is not a zero divisor) that under the equivalence (2.9) the operation $F \otimes$ ? corresponds to the operation $\left.F\right|_{X_{0}} \otimes$ ? on the singularity category $D_{\mathrm{Sg}, \mathbb{G}_{m}}\left(X_{0}\right)$.

Assume now in addition that the action of $\mathbb{G}_{m}$ on $X$ is trivial and $X$ is quasiprojective. Then we can define several complexes associated with a $\mathbb{G}_{m}$-equivariant matrix factorization of 0 on $X$. Note that for any $\mathbb{G}_{m}$-equivariant matrix factorization $E$ of 0 we can write $E_{0}=\bigoplus E_{0, i}, E_{1}=\bigoplus E_{1, i}$, where $\mathbb{G}_{m}$ acts on $E_{\bullet, i}$ through the character $\lambda \mapsto \lambda^{-i}$, i.e., $E_{\bullet}, i=\left(E_{\bullet}\{i\}\right)^{\mathbb{G}_{m}}$. Then we have the functor

$$
\operatorname{com}_{0}: \mathrm{DMF}_{\mathbb{G}_{m}, d}(X, 0) \rightarrow \operatorname{Per}(X),
$$

where $\operatorname{com}_{0}(E)$ is the complex

$$
\ldots \rightarrow E_{1,0} \rightarrow E_{0,0} \rightarrow E_{1, d} \rightarrow \ldots
$$

with $E_{0,0}$ placed in degree 0 . Note that $E_{0, i}$ and $E_{1, i}$ are nonzero only for finitely many $i$, so the complex $\operatorname{com}_{0}(E)$ is bounded. Let us also consider the functor

$$
\overline{\mathrm{com}}: \operatorname{DMF}_{\mathbb{G}_{m}, d}(X, 0) \rightarrow \operatorname{Per}(X), \overline{\operatorname{com}}(E)=\bigoplus_{i=0}^{d-1} \operatorname{com}_{0}(E\{i\}) .
$$


Proposition 2.5.3. Let $X$ be a scheme equipped with the trivial $\mathbb{G}_{m}$-action.

(i) For $E \in \mathrm{DMF}_{\mathbb{G}_{m}, d}(X, 0)$ one has a natural isomorphism of complexes

$$
\operatorname{com}_{0}(E) \simeq \operatorname{com}(E)^{\mathbb{G}_{m}}
$$

(ii) The functor mf is left adjoint to $\mathrm{com}_{0}$.

(iii) The left adjoint to the functor $\overline{\mathrm{com}}$ is $F \mapsto \bigoplus_{i=0}^{d-1} \operatorname{mf}(F)\{-i\}$.

(iv) For $F \in \operatorname{Per}(X)$ and $E \in \mathrm{DMF}_{\mathbb{G}_{m}, d}(X, 0)$ one has a natural isomorphism of complexes

$$
\overline{\operatorname{com}}(F \otimes E) \simeq F \otimes \overline{\operatorname{com}}(E)
$$

where on the left we equip $F$ with the trivial $\mathbb{G}_{m}$-action.

Proof. (i) This follows immediately from the definitions.

(ii) For a complex $C^{\bullet} \in \operatorname{Per}(X)$ and a matrix factorization $F$ let us compute the complex $\underline{\operatorname{Hom}}\left(\operatorname{mf}\left(C^{\bullet}\right), F\right)^{\mathbb{G}_{m}}$. We have

$$
\begin{aligned}
& \underline{\operatorname{Hom}}^{2 n}\left(\operatorname{mf}\left(C^{\bullet}\right), F\right)^{\mathbb{G}_{m}}=\underline{\operatorname{Hom}}\left(\bigoplus_{i} C^{2 i}\{-i d\}, F_{0}\{d n\}\right)^{\mathbb{G}_{m}} \oplus \underline{\operatorname{Hom}}\left(\bigoplus_{n} C^{2 i-1}\{-i d\}, F_{1}\{d n\}\right)^{\mathbb{G}_{m}}= \\
& \prod_{i} \underline{\operatorname{Hom}}\left(C^{2 i}, F_{0, d(i+n)}\right) \oplus \prod_{i} \underline{\operatorname{Hom}}\left(C^{2 i-1} F_{1, d(i+n)}\right)=\prod_{j} \underline{\operatorname{Hom}}\left(C^{j}, \operatorname{com}_{0}(F)^{j+2 n}\right) .
\end{aligned}
$$

Similarly

$$
\underline{\operatorname{Hom}}^{2 n+1}\left(\operatorname{mf}\left(C^{\bullet}\right), F\right)^{\mathbb{G}_{m}}=\prod_{j} \underline{\operatorname{Hom}}\left(C_{j}, \operatorname{com}_{0}(F)^{j+2 n+1}\right),
$$

and the differentials match. Passing to $R \Gamma(X$, ?) we get the required adjointness.

(iii) This follows easily from (ii).

(iv) This follows from (2.12): first, we check a similar property for $\mathrm{com}_{0}$, and then for $\overline{\mathrm{com}}$.

Remark 2.5.4. When the action of $\mathbb{G}_{m}$ on $X$ is trivial, we have an equivalence

$$
\operatorname{DMF}_{\mathbb{G}_{m}, d}(X, 0) \simeq \operatorname{Per}_{\mu_{d}}(X)
$$

associating to $E$ the complex $\bigoplus_{i=0}^{d-1} \operatorname{com}_{0}(E\{i\})\{-i\}$ (cf. [36, Prop. 1.2.2]). The functor $\overline{\mathrm{com}}$ is the composition of this equivalence with the forgetful functor $\operatorname{Per}_{\mu_{d}}(X) \rightarrow \operatorname{Per}(X)$.

Let us now return to the general situation of a $\mathbb{G}_{m}$-scheme $X$ with a function $W$ of weight $d>0$. For a closed $\mathbb{G}_{m}$-invariant subset $T \subset X_{0}=W^{-1}(0)$ we denote by $\operatorname{DMF}_{\mathbb{G}_{m}, T}(X, W) \subset$ $\mathrm{DMF}_{\mathbb{G}_{m}}(X, W)$ the full subcategory of matrix factorizations $E$ such that for every closed point $x \in X_{0} \backslash T$ the complex $\left.\operatorname{com}(E)\right|_{x}$ is exact. Equivalently, these are matrix factorizations that become trivial in the category $\operatorname{DMF}_{\mathbb{G}_{m}}(X \backslash T, W)$ (see [35, Lem. 5.4(iii)]).

Let $f: X \rightarrow Y$ be a $\mathbb{G}_{m}$-morphism of smooth $\mathbb{G}_{m}$-varieties, and let $W$ be a function of weight $d>0$ on $Y$. The push-forward functor $f_{*}$ for matrix factorizations is most naturally 
defined in terms of matrix factorizations of quasicoherent sheaves (see [15, Sec. 3], [2]). If $T \subset X$ is a closed subset, proper over $Y$, then one has also a natural push-forward functor

$$
f_{*}: \overline{\mathrm{DMF}}_{\mathbb{G}_{m}, T}\left(X, f^{*} W\right) \rightarrow \overline{\mathrm{DMF}}_{\mathbb{G}_{m}}(Y, W)
$$

(see [15, Sec. 3.4], [35, Sec. 6]).

Assume in addition that $W$ and $f^{*} W$ are not zero divisors. Let $f_{0}: X_{0}=f^{-1}\left(W^{-1}(0)\right) \rightarrow$ $Y_{0}=W^{-1}(0)$ be the morphism between the hypersurfaces induced by $f$. Then the functor $f_{0 *}: D^{b}\left(X_{0}\right) \rightarrow D^{b}\left(Y_{0}\right)$ induces a functor between the singularity categories, which corresponds to $f_{*}$ under the equivalences (2.9) for $W$ and $f^{*}(W)$.

The following property is straightforward.

Lemma 2.5.5. For $f: X \rightarrow Y$ a $\mathbb{G}_{m}$-equivariant morphism of smooth $\mathbb{G}_{m}$-varieties, and for $F, a \mathbb{G}_{m}$-equivariant matrix factorization of 0 on $X$, one has

$$
\operatorname{com}\left(f_{*}(F)\right) \simeq f_{*} \operatorname{com}(F) .
$$

The analog of the Grothendieck duality for matrix factorizations was established under quite general assumptions in the work of Efimov and Positselski [15, Sec. 3]. Here we will use the following version for smooth morphisms between smooth varieties.

Proposition 2.5.6. Let $f: X \rightarrow Y$ be a smooth $\mathbb{G}_{m}$-equivariant morphism of relative dimension $m$ between smooth $\mathbb{G}_{m}$-varieties, and let $T \subset X$ be a closed subset, proper over $Y$. Let $W$ be a function on $Y$ of weight $d>0$ with respect to the $\mathbb{G}_{m}$-action, which is not a zero divisor. For $E \in \overline{\mathrm{DMF}}_{\mathbb{G}_{m}, T}\left(X, f^{*} W\right)$ we have a natural functorial isomorphism

$$
f_{*}\left(E^{\vee} \otimes \mathcal{D}_{f}\right) \simeq\left(f_{*} E\right)^{\vee}
$$

in $\overline{\mathrm{DMF}}_{\mathbb{G}_{m}}(Y,-W)$, where

$$
\mathcal{D}_{f}:=\omega_{f}[m] .
$$

Proof. Let $f_{0}: X_{0} \rightarrow Y_{0}=W^{-1}(0)$ be the morphism induced by $f$, where $X_{0}=f^{-1}\left(Y_{0}\right)$. Note that under the equivalence (2.9), extended to Caroubian completions, $E$ corresponds to an object $F \in \bar{D}_{\mathrm{Sg}}\left(X_{0}\right)$ supported at $T \cap X_{0}$ (see [35, Prop. 5.6]). Hence, by Lemma 2.5.1, it suffices to construct an isomorphism

$$
f_{0 *}\left(\left.R \underline{\operatorname{Hom}}\left(F, \mathcal{O}_{X_{0}}\right) \otimes \mathcal{D}_{f}\right|_{X_{0}}\right) \simeq R \underline{\operatorname{Hom}}\left(f_{0 *} F, \mathcal{O}_{Y_{0}}\right)
$$

in $D^{b}\left(Y_{0}\right)$, provided $F \in D^{b}\left(X_{0}\right)$ has a support proper over $Y_{0}$. Since $\left.\mathcal{D}_{f}\right|_{X_{0}} \simeq \mathcal{D}_{f_{0}}$, such an isomorphism is given by the usual Grothendieck duality.

Corollary 2.5.7. In the situation of Proposition [2.5.6, for $E \in \overline{\mathrm{DMF}}_{\mathbb{G}_{m}, T}\left(X, f^{*} W\right)$ and $F \in \overline{\mathrm{DMF}}_{\mathbb{G}_{m}}(Y, W)$ one has

$$
\begin{gathered}
\underline{\operatorname{Hom}}\left(f_{*} E, F\right) \simeq f_{*} \underline{\operatorname{Hom}}\left(E, f^{+} F\right), \text { where } \\
f^{+} F=\mathcal{D}_{f} \otimes f^{*} F .
\end{gathered}
$$

Hence, we also have a functorial isomorphism

$$
\operatorname{Hom}\left(f_{*} E, F\right) \simeq \operatorname{Hom}\left(E, f^{+} F\right) .
$$


Proof. Using Lemma 2.5.2, Proposition [2.5.6, and the projection formula we get

$$
\underline{\operatorname{Hom}}\left(f_{*} E, F\right) \simeq \operatorname{com}\left(\left(f_{*} E\right)^{\vee} \otimes F\right) \simeq \operatorname{com}\left(f_{*}\left(E^{\vee} \otimes \mathcal{D}_{f} \otimes f^{*} F\right)\right) .
$$

Using Lemma 2.5.5 we can switch com with $f_{*}$, so we obtain

$$
\underline{\operatorname{Hom}}\left(f_{*} E, F\right) \simeq f_{*} \operatorname{com}\left(E^{\vee} \otimes \mathcal{D}_{f} \otimes f^{*} F\right) \simeq f_{*} \underline{\operatorname{Hom}}\left(E, \mathcal{D}_{f} \otimes f^{*} F\right),
$$

as claimed. The second isomorphism is obtained from the first by applying $R \Gamma$.

Let us recall an important construction of Koszul matrix factorizations, which can be viewed as a generalization of the Koszul complex. Assume we have a $\mathbb{G}_{m}$-equivariant vector bundle $V$ on $X$ and invariant global sections

$$
\alpha \in H^{0}(X, V\{d\})^{\mathbb{G}_{m}}, \beta \in H^{0}\left(X, V^{\vee}\right)^{\mathbb{G}_{m}} \text { such that }\langle\alpha, \beta\rangle=W .
$$

Then we define the Koszul matrix factorization $\{\alpha, \beta\}$ of $W$ by

$$
\begin{gathered}
\{\alpha, \beta\}_{0}=\mathcal{O}_{X} \oplus \wedge^{2} V\{d\} \oplus \wedge^{4} V\{2 d\} \oplus \ldots, \\
\{\alpha, \beta\}_{1}=V \oplus \wedge^{3} V\{d\} \oplus \wedge^{5} V\{2 d\} \oplus \ldots,
\end{gathered}
$$

with the differential given by

$$
\delta_{\alpha, \beta}=\alpha \wedge ?+\iota_{\beta},
$$

where $\iota_{\beta}$ is the contraction by $\beta$. An important fact is that $\{\alpha, \beta\}$ is supported on the locus of common zeros of $\alpha$ and $\beta$ (see [36, Lem. 1.5.1]).

\subsection{Some functors given by kernels and an adjunction between them}

Suppose we have a diagram of smooth $\mathbb{G}_{m}$-varieties

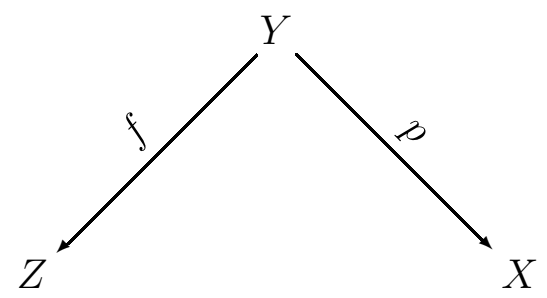

where $f$ is a smooth morphism of constant relative dimension, and $\mathbb{G}_{m}$ acts trivially on $X$. Assume further that $W$ is a function on $Z$ of weight $d>0$ with respect to the $\mathbb{G}_{m}$-action. Given a $\mathbb{G}_{m}$-equivariant matrix factorization $P$ of $-f^{*} W$ on $Y$, with proper support, we can define functors

$$
\begin{aligned}
& \widetilde{\Phi}_{P}: \overline{\mathrm{DMF}}_{\mathbb{G}_{m}}(Z, W) \rightarrow \overline{\mathrm{DMF}}_{\mathbb{G}_{m}, d}(X, 0), \quad \Phi_{P}: \overline{\mathrm{DMF}}_{\mathbb{G}_{m}}(Z, W) \rightarrow D^{b}(X),
\end{aligned}
$$

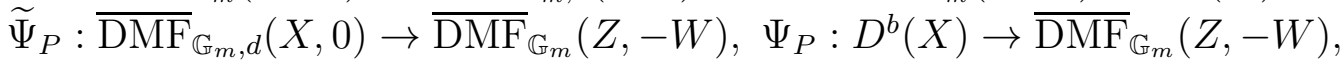


as follows:

$$
\begin{gathered}
\widetilde{\Phi}_{P}(E)=p_{*}\left(f^{*} E \otimes P\right), \Phi_{P}=\overline{\operatorname{com}} \circ \widetilde{\Phi}_{P}, \\
\widetilde{\Psi}_{P}(F)=f_{*}\left(P \otimes p^{*} F\right), \quad \Psi_{P}(F)=\bigoplus_{i=0}^{d-1} \widetilde{\Psi}_{P}(\operatorname{mf}(F)\{-i\}) .
\end{gathered}
$$

Here $f^{*} E \otimes P\left(\right.$ resp., $\left.P \otimes p^{*} F\right)$ is a $\mathbb{G}_{m^{-}}$-equivariant matrix factorization of 0 (resp., $-f^{*} W$ ) on $Y$, that has a proper support, and so we can apply the push-forward functor $p_{*}$ (resp., $f_{*}$ ) to it. The functors $\mathrm{mf}$ and $\overline{\mathrm{com}}$ are given by (2.11) and (2.13).

Lemma 2.6.1. Let

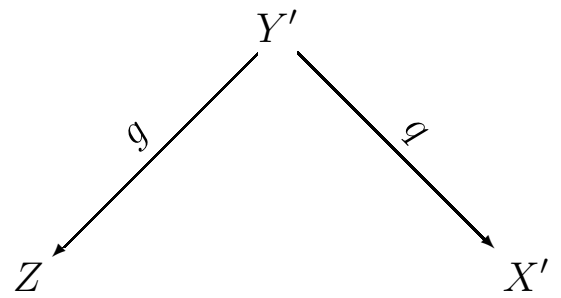

be another diagram with the same properties as (2.15), and let $P^{\prime}$ be a $\mathbb{G}_{m}$-equivariant matrix factorization of $g^{*} W$ on $Y^{\prime}$ with proper support. Then the composition

$$
\overline{\mathrm{DMF}}_{\mathbb{G}_{m}, d}\left(X^{\prime}, 0\right) \stackrel{\widetilde{\Psi}_{P^{\prime}}}{\longrightarrow} \overline{\mathrm{DMF}}_{\mathbb{G}_{m}}(Z, W) \stackrel{\widetilde{\Phi}_{P}}{\longrightarrow} \overline{\mathrm{DMF}}_{\mathbb{G}_{m}, d}(X, 0)
$$

is isomorphic to the Fourier-Mukai type functor associated with the kernel

$$
\widetilde{K}=p_{X^{\prime} X, *}\left(p_{1}^{*} P^{\prime} \otimes p_{2}^{*} P\right) \in{\overline{\mathrm{DMF}_{\mathbb{G}_{m}, d}}}\left(X^{\prime} \times X, 0\right),
$$

where we consider the diagram

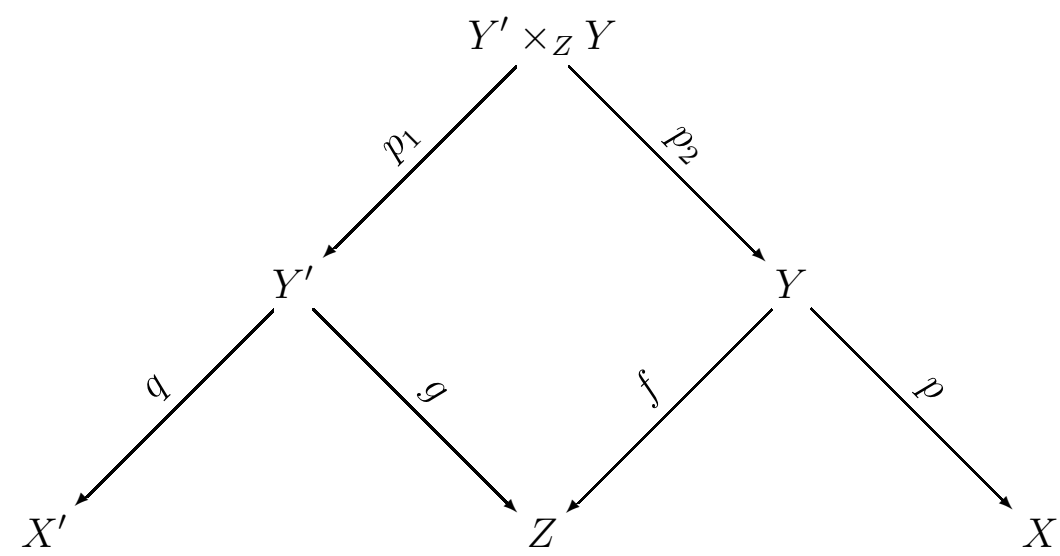

and denote by $p_{X^{\prime} X}: Y^{\prime} \times_{Z} Y \rightarrow X^{\prime} \times X$ the map induced by $q \circ p_{1}$ and $p \circ p_{2}$. The composition

$$
\Phi_{P} \circ \Psi_{P^{\prime}}: D^{b}\left(X^{\prime}\right) \rightarrow D^{b}(X)
$$

is isomorphic to the Fourier-Mukai functor associated with a kernel $K \in D^{b}\left(X^{\prime} \times X\right)$ such that $[K]=d[\overline{\operatorname{com}}(\widetilde{K})]$ in $K_{0}\left(X^{\prime} \times X\right)$. 
Proof. The first assertion follows easily from the projection formula and the base change formula (cf. [2, Sec. 5.2]). It remains to compute the composition

$$
\Phi_{P} \circ \Psi_{P^{\prime}}: F \mapsto \overline{\operatorname{com}}\left(\widetilde{\Phi}_{P} \circ \widetilde{\Psi}_{P^{\prime}}\right)\left(\bigoplus_{i=0}^{d-1} \operatorname{mf}(F)\{-i\}\right)
$$

Let $p_{X}: X^{\prime} \times X \rightarrow X, p_{X^{\prime}}: X^{\prime} \times X \rightarrow X^{\prime}$ be the projections. We have

$$
\overline{\operatorname{com}}\left(p_{X, *}\left(p_{X^{\prime}}^{*} \operatorname{mf}(F)\{-i\} \otimes \widetilde{K}\right)\right) \simeq p_{X, *} \overline{\operatorname{com}}\left(p_{X^{\prime}}^{*} F \otimes \widetilde{K}\{-i\}\right) \simeq p_{X, *}\left(p_{X^{\prime}}^{*} F \otimes \overline{\operatorname{com}}(\widetilde{K}\{-i\})\right)
$$

where in the last isomorphism we used Proposition 2.5.3(iv). Thus, $\Phi_{P} \circ \Psi_{P^{\prime}}$ is associated with the kernel

$$
K=\bigoplus_{i=0}^{d-1} \overline{\operatorname{com}}(\widetilde{K}\{-i\})
$$

It is easy to see that $\overline{\operatorname{com}}(\widetilde{K}\{-i\})$ and $\overline{\operatorname{com}}(\widetilde{K})$ are direct sums of almost the same complexessome complexes get shifted by even integers. Hence, the assertion about the classes in the Grothendieck group.

As before, we denote $\mathcal{D}_{f}=\omega_{f}[\operatorname{dim} Y-\operatorname{dim} Z]$.

Proposition 2.6.2. In the above situation the left adjoint functor to $\widetilde{\Phi}_{P}$ (resp., $\Phi_{P}$ ) is $\widetilde{\Psi}_{Q}\left(\right.$ resp., $\left.\Psi_{Q}\right)$, where $Q=P^{\vee} \otimes \mathcal{D}_{f} \in \mathrm{MF}_{\mathbb{G}_{m}}\left(Y, f^{*} W\right)$. Furthermore, the corresponding dg-functors are adjoint in the sense of Definition 2.1.1.

Proof. For $E \in \mathrm{MF}_{\mathbb{G}_{m}, d}(X, 0)$ and $F \in \mathrm{MF}_{\mathbb{G}_{m}}(Z, W)$, we have a chain of quasi-isomorphisms

$$
\begin{aligned}
& \operatorname{Hom}\left(E, \widetilde{\Phi}_{P}(F)\right) \simeq \operatorname{Hom}\left(E, p_{*}\left(P \otimes f^{*} F\right)\right) \simeq \\
& \operatorname{Hom}\left(p^{*} E, P \otimes f^{*} F\right) \simeq \operatorname{Hom}\left(p^{*} E \otimes P^{\vee} \otimes \mathcal{D}_{f}, f^{*} F \otimes \mathcal{D}_{f}\right) .
\end{aligned}
$$

Note that $p^{*} E \otimes P^{\vee} \otimes \mathcal{D}_{f}$ has proper support. Hence, using Corollary 2.5.7 we can rewrite this as

$$
\operatorname{Hom}\left(f_{*}\left(p^{*} E \otimes P^{\vee} \otimes \mathcal{D}_{f}\right), F\right) \simeq \operatorname{Hom}\left(\widetilde{\Psi}_{Q}(E), F\right) .
$$

To show the required adjunction at the dg-level we have to prove that the above isomorphism is induced by a map Id $\rightarrow \widetilde{\Phi}_{P} \circ \widetilde{\Psi}_{Q}$ given by a map of kernels. By Lemma 2.6.1, the composition $\widetilde{\Phi}_{P} \circ \widetilde{\Psi}_{Q}$ is the functor given by the kernel

$$
p_{X X, *}\left(p_{1}^{*} Q \otimes p_{2}^{*} P\right) \in \mathrm{MF}_{\mathbb{G}_{m}, d}\left(X^{\prime} \times X, 0\right),
$$

where $p_{1}$ and $p_{2}$ are the two projections $Y \times_{Z} Y \rightarrow Y$, and

$$
p_{X X}: Y \times_{Z} Y \rightarrow X \times X
$$

is the map with the components $\left(p p_{1}, p p_{2}\right)$. Thus, we need a map of kernels

$$
\Delta_{*} \mathcal{O}_{X} \rightarrow p_{X X, *}\left(p_{1}^{*} Q \otimes p_{2}^{*} P\right) .
$$


The construction of this map and the verification that the corresponding natural transformation $\operatorname{Id}_{\mathrm{MF}_{\mathbb{G}_{m}, d}(X, 0)} \rightarrow \widetilde{\Phi}_{P} \circ \widetilde{\Psi}_{Q}$ induces the same quasi-isomorphism

$$
\operatorname{Hom}\left(\widetilde{\Psi}_{Q}(E), F\right) \stackrel{\sim}{\longrightarrow} \operatorname{Hom}\left(E, \widetilde{\Phi}_{P}(F)\right)
$$

as the one obtained above are done in the Appendix (see (5.8) and Proposition 5.0.3).

The adjunction of the pair $\left(\Psi_{Q}, \Phi_{P}\right)$ follows from the adjunction of the pair $\left(\widetilde{\Psi}_{Q}, \widetilde{\Phi}_{P}\right)$ using Proposition 2.5.3(iii).

Now assume that in the above situation we have $Z=\mathbb{A}^{n}$ and $W$ is a quasihomogeneous polynomial on $Z$ of degree $d>0$ with an isolated singularity. Recall that we have a decomposition

$$
H H_{*}\left(\mathrm{MF}_{\mathbb{G}_{m}}(W)\right)=\mathcal{H}(W)=\bigoplus_{\gamma \in \mu_{d}} H\left(W_{\gamma}\right)^{\mu_{d}}
$$

where $W_{\gamma}=\left.W\right|_{\left(\mathbb{A}^{n}\right)^{\gamma}}$ (see [36, Thm. 2.6.1]). In fact, this decomposition is exactly the $\mu_{d^{-}}$ grading associated with the natural $\mathbb{Z} / d$-action on $H H_{*}\left(\mathrm{MF}_{\mathbb{G}_{m}}(W)\right)$ induced by the functors $E \mapsto E\{i\}$ (see [36, Thm. 2.6.1(ii)]). Let $\Pi$ denote the projector of $\mathcal{H}(W)$ onto the summand $H(W)^{\mu_{d}}$, corresponding to $\gamma=1$.

Lemma 2.6.3. Let $\phi_{P}: \mathcal{H}(W) \rightarrow H H_{*}(X)=H^{*}(X, \mathbb{C})$ be the map induced on Hochschild homology by the functor $\Phi_{P}$ given by (2.16). We have $\phi_{P}=\phi_{P} \circ \Pi$.

Proof. It is clear from the definition that the functor $\widetilde{\Phi}_{P}$ commutes with tensoring by characters of $\mathbb{G}_{m}$. Hence, $\Phi_{P}(F) \simeq \Phi_{P}(F\{i\})$. It follows that the map $\phi_{P}$ is $\mathbb{Z} / d$-invariant, which is equivalent to the equality in question.

\subsection{Orlov's equivalence}

Now let $W\left(x_{1}, \ldots, x_{n}\right)$ be a homogeneous polynomial of degree $d>0\left(\operatorname{so} \operatorname{deg}\left(x_{i}\right)=1\right)$ with an isolated singularity. Let us recall Orlov's construction in [29, Sec. 2.1, 2.2] relating the category of $\mathbb{G}_{m}$-equivariant matrix factorizations of $W$ with the derived category of coherent sheaves on the smooth projective hypersurface $Y \subset \mathbb{P}^{n-1}$ given by the equation $W=0$.

The construction proceeds in several steps. First, consider the graded algebra

$$
A=k\left[x_{1}, \ldots, x_{n}\right] /(W) .
$$

Note that the algebra $A$ is Gorenstein with the Gorenstein parameter $a=n-d$, i.e., $\operatorname{Ext}_{A}^{*}(k, A)$ is concentrated in internal degree $a$ (and in cohomological degree $n-1$ ). We denote by gr $-A$ the category of finitely generated graded $A$-modules, and by $\mathrm{gr}-A_{\geq i}$ the full subcategory of modules $M$ with $M_{j}=0$ for $j<i$.

We have Serre's description of coherent sheaves on $Y=\operatorname{Proj}(A)$ as the quotient

$$
\operatorname{qgr} A=\operatorname{gr}-A / \text { tors }-A
$$


of $\mathrm{gr}-A$ by the subcategory of torsion modules. Thus, we have an equivalence

$$
D^{b}(Y) \simeq D^{b}(\operatorname{qgr} A) .
$$

Note that under this equivalence the sheaf $\mathcal{O}_{Y}(i)$ corresponds to $A(i) \in$ qgr $A$.

Next, for each $i \in \mathbb{Z}$ we have a fully faithful functor

$$
R \omega_{i}: D^{b}(\operatorname{qgr} A) \rightarrow D^{b}\left(\operatorname{gr}-A_{\geq i}\right) \subset D^{b}(\operatorname{gr}-A),
$$

which is right adjoint to the natural projection $\pi_{i}: D^{b}\left(\operatorname{gr}-A_{\geq i}\right) \rightarrow D^{b}(\operatorname{qgr} A)$. The image of $R \omega_{i}$ is denoted by $\mathcal{D}_{i}$. The natural projection

$$
\pi: D^{b}(\operatorname{gr}-A) \rightarrow D^{b}(\operatorname{qgr} A) \simeq D^{b}(Y)
$$

induces an equivalence $\mathcal{D}_{i} \simeq D^{b}(Y)$.

Recall that a full triangulated subcategory $\mathcal{T}^{\prime}$ of a triangulated category $\mathcal{T}$ is called left (resp., right) admissible if the inclusion functor $\mathcal{T}^{\prime} \rightarrow \mathcal{T}$ has a left (resp., right) adjoint functor $\mathcal{T} \rightarrow \mathcal{T}^{\prime}$. A subcategory is admissible if it is left and right admissible. A semiorthogonal decomposition

$$
\mathcal{T}=\left\langle\mathcal{A}_{1}, \ldots, \mathcal{A}_{r}\right\rangle
$$

is given by a collection of full triangulated subcategories $\left(\mathcal{A}_{i}\right)$ such that there exists an increasing filtration $0=\mathcal{T}_{0} \subset \mathcal{T}_{1} \subset \ldots \subset \mathcal{T}_{r}=\mathcal{T}$ by left admissible subcategories such that $\mathcal{A}_{i}$ is the left orthogonal of $\mathcal{T}_{i-1}$ in $\mathcal{T}_{i}$. In particular, $\operatorname{Hom}_{\mathcal{J}}\left(\mathcal{A}_{j}, \mathcal{A}_{i}\right)=0$ for $j>i$ and $\left(\mathcal{A}_{i}\right)$ generate $\mathcal{T}$ as a triangulated category. Note that if in addition $\mathcal{T}=D^{b}(X)$, where $X$ is a smooth projective variety, then each $\mathcal{A}_{i}$ is an admissible subcategory in $\mathcal{T}$ (see [5]). When one of the subcategories $\mathcal{A}_{i}$ is generated by an exceptional object $A_{i}$ then we write simply $A_{i}$ instead of $\mathcal{A}_{i}$ in the right-hand side of (2.18).

Let $\mathcal{P}_{\geq i}$ (resp., $\left.\mathcal{S}_{<i}\right)$ denote the triangulated subcategory of $D^{b}(\mathrm{gr}-A)$ generated by $A(e)$ with $e \leq-i$ (resp., by $k(e)$ with $e>i$ ). Then we have semiorthogonal decompositions

$$
\begin{gathered}
D^{b}(\operatorname{gr}-A)=\left\langle\mathcal{S}_{<i}, D^{b}\left(\operatorname{gr}-A_{\geq i}\right)\right\rangle, \\
D^{b}\left(\operatorname{gr}-A_{\geq i}\right)=\left\langle\mathcal{P}_{\geq i}, \mathcal{T}_{i}\right\rangle,
\end{gathered}
$$

where $\mathcal{T}_{i}$ is equivalent via the natural projection from $D^{b}\left(\mathrm{gr}-A_{\geq i}\right)$ to the graded singularity category of $A$, which in turn is equivalent to the homotopy category of graded matrix factorizations (by [29, Thm. 3.10]). Combining these two decompositions we get a semiorthogonal decomposition

$$
D^{b}(\mathrm{gr}-A)=\left\langle\mathcal{S}_{<i}, \mathcal{P}_{\geq i}, \mathcal{T}_{i}\right\rangle .
$$

On the other hand, there is a semiorthogonal decomposition

$$
D^{b}(\mathrm{gr}-A)=\left\langle\mathcal{P}_{\geq i+a}, \mathcal{S}_{<i}, \mathcal{D}_{i}\right\rangle,
$$

where $a=n-d$. 
For $a=0$ the above decompositions for $i=0$ imply that $\mathcal{D}_{0}=\mathcal{T}_{0}$, and hence we get an equivalence relating matrix factorizations and coherent sheaves on $Y$ :

$$
\mathrm{DMF}_{\mathbb{G}_{m}}(W) \simeq \mathcal{T}_{0}=\mathcal{D}_{0} \simeq D^{b}(Y)
$$

More generally, for $a \geq 0$ we can use the semiorthogonal decomposition

$$
\mathcal{P}_{\geq i}=\left\langle\mathcal{P}_{\geq i+a}, A(-i-a+1), \ldots, A(-i-1), A(-i)\right\rangle
$$

to refine the decomposition $(2.19)$ to

$$
D^{b}(\operatorname{gr}-A)=\left\langle\mathcal{S}_{<i}, \mathcal{P}_{\geq i+a}, A(-i-a+1), \ldots, A(-i-1), A(-i), \mathcal{T}_{i}\right\rangle .
$$

Comparing this with (2.20) we get a semiorthogonal decomposition

$$
\mathcal{D}_{i}=\left\langle A(-i-a+1), \ldots, A(-i-1), A(-i), \mathcal{T}_{i}\right\rangle .
$$

Thus, in this case the category $D^{b}(Y)$ contains $\operatorname{DMF}_{\mathbb{G}_{m}}(W)$ as an admissible subcategory. Let

$$
\rho: D^{b}(Y) \simeq \mathcal{D}_{0} \rightarrow \mathcal{T}_{0} \simeq \mathrm{DMF}_{\mathbb{G}_{m}}(W)
$$

be the right adjoint functor to the embedding $\mathcal{T}_{0} \subset \mathcal{D}_{0}$.

In the case $a \leq 0$, using the decomposition

$$
\mathcal{S}_{<-a}=\left\langle\mathcal{S}_{<0}, k, k(-1), \ldots, k(a+1)\right\rangle,
$$

we refine the decomposition (2.20) for $i=-a$ to

$$
D^{b}(\operatorname{gr}-A)=\left\langle\mathcal{P}_{\geq 0}, \mathcal{S}_{<0}, k, k(-1), \ldots, k(a+1), \mathcal{D}_{-a}\right\rangle .
$$

Comparing this with (2.19) for $i=0$ we get a semiorthogonal decomposition

$$
\mathcal{T}_{0}=\left\langle k, k(-1), \ldots, k(a+1), \mathcal{D}_{-a}\right\rangle .
$$

Thus, in this case $\operatorname{DMF}_{\mathbb{G}_{m}}(W)$ contains $D^{b}(Y)$ as a full subcategory. Let

$$
\rho: D^{b}(Y) \simeq \mathcal{D}_{-a} \rightarrow \mathcal{T}_{0} \simeq \operatorname{DMF}_{\mathbb{G}_{m}}(W)
$$

be the corresponding fully faithful functor.

Caldararu and Tu showed that the above constructions can be performed at the dg-level (see [7, Sec. 5]). In particular, the functors (2.22) and (2.24) lift to the dg-level.

For any graded module $M$ over $A$ we denote by $M^{\text {st }}$ the object of $\operatorname{DMF}_{\mathbb{G}_{m}}(W)$ corresponding to $M$ viewed as an object of the graded singularity category.

Proposition 2.7.1. Let us consider the composition

$$
\Phi: D^{b}\left(\mathbb{P}^{n-1}\right) \rightarrow D^{b}(Y) \stackrel{\rho}{\longrightarrow} \mathcal{T}_{0} \simeq \operatorname{DMF}_{\mathbb{G}_{m}}(W),
$$

where the first arrow is the pull-back with respect to the embedding $Y \subset \mathbb{P}^{n-1}$, and $\rho$ is given by (2.22) for $a \geq 0$ and by (2.24) for $a \leq 0$. Then the image of $\Phi$ is contained in the triangulated subcategory of $\mathrm{DMF}_{\mathbb{G}_{m}}(W)$, generated by $\left(k(-e)^{\mathrm{st}}\right)_{e \in \mathbb{Z}}$. 
Proof. Assume first that $a \leq 0$. For $e<0$ let us consider the truncated module $A(-e)_{\geq-a}$, so that we have an exact sequence

$$
0 \rightarrow A(-e)_{\geq-a} \rightarrow A(-e) \rightarrow N_{e} \rightarrow 0,
$$

where $N_{e} \in\langle k(-e), k(-e-1), \ldots, k(a+1)\rangle$. Then we claim that $A(-e)_{\geq-a} \in \mathcal{D}_{-a}$. Indeed, we have $A(-e)_{\geq-a} \in D^{b}\left(\mathrm{gr}-A_{\geq-a}\right)$, so by the decomposition (2.20), it is enough to check that

$$
\operatorname{Ext}_{\mathrm{gr}-A}^{*}\left(A(-e)_{\geq-a}, \mathcal{P}_{\geq 0}\right)=0 .
$$

But this follows from the exact sequence (2.25), since $\operatorname{Ext}_{\mathrm{gr}-A}^{*}\left(k(-j), \mathcal{P}_{\geq 0}\right)=0$ for $j<-a$. Now the same exact sequence shows that on the one hand,

$$
\pi\left(A(-e)_{\geq-a}\right)=\mathcal{O}(-e),
$$

while on the other hand, the image of $A(-e)_{\geq-a}$ in the graded singularity category is the same as that of $N_{e}[-1]$. Hence, we deduce that

$$
\Phi(\mathcal{O}(-e)) \in\left\langle k(-e)^{\mathrm{st}}, k(-e-1)^{\mathrm{st}}, \ldots, k(a+1)^{\mathrm{st}}\right\rangle \subset \mathrm{DMF}_{\mathbb{G}_{m}}(W) .
$$

Since $D^{b}\left(\mathbb{P}^{n-1}\right)$ is generated by the sheaves $\mathcal{O}(-e)$ with $e<0$, the assertion follows in this case.

Now assume that $a \geq 0$. Then for $e<0$ we consider the truncated module $A(-e)_{\geq 0}$ that fits into an exact sequence

$$
0 \rightarrow A(-e)_{\geq 0} \rightarrow A(-e) \rightarrow M_{e} \rightarrow 0
$$

where $M_{e} \in\langle k(-e), \ldots, k(1)\rangle$. Then as above we deduce that $A(-e)_{\geq 0} \in \mathcal{D}_{0}$ and

$$
\pi\left(A(-e)_{\geq 0}\right)=\mathcal{O}(-e) .
$$

On the other hand, by the semiorthogonal decomposition (2.21) for $i=0$, we have an exact triangle

$$
\rho\left(A(-e)_{\geq 0}\right) \rightarrow A(-e)_{\geq 0} \rightarrow Q \rightarrow \ldots
$$

with $Q \in\langle A(-a+1), \ldots, A(-1), A\rangle$. It follows that the image of $\rho\left(A(-e)_{\geq 0}\right)$ in the graded singularity category is the same as that of $M_{e}$, so we deduce that

$$
\Phi(\mathcal{O}(-e)) \in\left\langle k(-e)^{\mathrm{st}}, \ldots, k(1)^{\mathrm{st}}\right\rangle \subset \mathrm{DMF}_{\mathbb{G}_{m}}(W) .
$$

\section{Rational structure on the Hochschild homology}

\subsection{Rational structure on the Hochschild homology of admissible subcategories in the derived categories of sheaves}

Let $X$ be a smooth projective variety over $\mathbb{C}$. Recall that the Hochschild homology $H H_{*}(X)$ can be identified with $H^{*}(X, \mathbb{C})=\bigoplus_{p, q} H^{p, q}(X)$ (with $H^{p, q}(X) \subset H H_{p-q}(X)$ ). We can 
use this identification to define a rational lattice in $H H_{*}(X)$. To get better compatibility with the Chern characters and Fourier-Mukai type functors we insert some standard factors. Namely, let us consider an automorphism

$$
J: H^{*}(X, \mathbb{C}) \rightarrow H^{*}(X, \mathbb{C}): c \mapsto(2 \pi i)^{p} c \quad \text { for } c \in H^{p, q}(X)
$$

and set2

$$
H H_{*}(X)_{\mathbb{Q}}:=J\left(H^{*}(X, \mathbb{Q})\right) \subset H^{*}(X, \mathbb{C}) \simeq H H_{*}(X)
$$

Proposition 3.1.1. (i) For any $E \in D^{b}(X)$ we have $\operatorname{ch}(E) \in H H_{*}(X)_{\mathbb{Q}}$.

(ii) For any $c, c^{\prime} \in H H_{*}(X)_{\mathbb{Q}}$ one has $\left(c, c^{\prime}\right)_{X} \in \mathbb{Q}$, where $(\cdot, \cdot)_{X}$ is the pairing (2.7).

(iii) For any Fourier-Mukai type functor $\Phi: D^{b}(X) \rightarrow D^{b}(Y)$, where $Y$ is smooth projective, the induced map $\Phi_{*}: H H_{*}(X) \rightarrow H H_{*}(Y)$ sends $H H_{*}(X)_{\mathbb{Q}}$ to $H H_{*}(Y)_{\mathbb{Q}}$.

Proof. (i) This follows immediately from Proposition 2.2.1.

(ii) We have

$$
\left(c, c^{\prime}\right)_{X}=\frac{1}{(2 \pi i)^{n}} \int_{[X]}^{t o p} c \cdot c^{\prime} \cdot \operatorname{Td}_{X},
$$

so the assertion follows from the fact that $c \cdot c^{\prime} \cdot \operatorname{Td}_{X} \in J\left(H^{*}(X, \mathbb{Q})\right)$.

(iii) This follows from (i) and (ii) and from Lemma 2.4.2.

We will use the real structure on $H H_{*}(X)=H^{*}(X, \mathbb{C})$ associated with the rational lattice $H H_{*}(X)_{\mathbb{Q}}=J\left(H^{*}(X, \mathbb{Q})\right)$. Let us denote by $\tau$ the corresponding complex conjugation map on $H^{*}(X, \mathbb{C}$ ), so that $\tau(J x)=J \bar{x}$ (where $x \mapsto \bar{x}$ is the usual complex conjugation on $\left.H^{*}(X, \mathbb{C})\right)$. It is easy to check that

$$
\tau(c)=(-1)^{p}(2 \pi i)^{q-p} \bar{c} \quad \text { for } c \in H^{p, q}(X) .
$$

Let $\mathcal{T} \subset D^{b}(X)$ be an admissible subcategory. We can enhance $\mathcal{T}$ to a dg-category using the dg-enhancement of $D^{b}(X)$ (see [23, Sec. 4]). By functoriality of the Hochschild homology, applied to the inclusion functor and to its left adjoint $D^{b}(X) \rightarrow \mathcal{T}$, the Hochschild homology $H H_{*}(\mathcal{T})$ gets identified with a direct summand of $H H_{*}(X)$.

Proposition 3.1.2. The subgroup $H H_{*}(\mathcal{T}) \cap H H_{*}(X)_{\mathbb{Q}}$ is a rational lattice in $H H_{*}(\mathcal{T})$. If $\mathcal{T} \hookrightarrow D^{b}\left(X^{\prime}\right)$ is a different embedding of $\mathcal{T}$ as admissible subcategory, where $X^{\prime}$ is a smooth projective variety, then

$$
H H_{*}(\mathcal{T}) \cap H H_{*}(X)_{\mathbb{Q}}=H H_{*}(\mathcal{T}) \cap H H_{*}\left(X^{\prime}\right)_{\mathbb{Q}},
$$

so that the lattice $H H_{*}(\mathcal{T})_{\mathbb{Q}}:=H H_{*}(\mathcal{T}) \cap H H_{*}(X)_{\mathbb{Q}}$ depends only on $\mathcal{T}$. If $\mathcal{T} \rightarrow \mathcal{T}^{\prime}$ is a dg-functor between two categories like this then the induced map $H H_{*}(\mathcal{T}) \rightarrow H H_{*}\left(\mathcal{T}^{\prime}\right)$ is compatible with these rational lattices.

\footnotetext{
${ }^{2}$ Choosing instead of $J$ the automorphism $c \mapsto(2 \pi i)^{q} c, c \in H^{p, q}(X)$, would work as well. The two choices differ by the grading operator with respect to the Hochschild degree.
} 
Proof. The projector functor $\Pi: D^{b}(X) \rightarrow \mathcal{T} \subset D^{b}(X)$ is given by some kernel (see [24, Thm. 7.1]). Hence, by Proposition 3.1.1, the induced projector $\Pi_{*}$ of $H H_{*}(X)$ with the image $H H_{*}(\mathcal{T})$ sends $H H_{*}(X)_{\mathbb{Q}}$ to $H H_{*}(\mathcal{T}) \cap H H_{*}(X)_{\mathbb{Q}}$, which implies that the latter subgroup is a rational lattice in $H H_{*}(\mathcal{T})$. Suppose we have a functor $\Phi: \mathcal{T} \rightarrow \mathcal{T}^{\prime}$, where $\mathcal{T}^{\prime} \subset D^{b}\left(X^{\prime}\right)$. Then by Proposition 3.1.1(iii), the composed map

$$
H H_{*}(X) \stackrel{\Pi_{*}}{\longrightarrow} H H_{*}(\mathcal{T}) \stackrel{\Phi_{*}}{\longrightarrow} H H_{*}\left(\mathcal{T}^{\prime}\right) \rightarrow H H_{*}\left(X^{\prime}\right)
$$

is compatible with rational lattices, hence, $\Phi_{*}$ sends $H H_{*}(\mathcal{T}) \cap H H_{*}(X)_{\mathbb{Q}}=\Pi_{*}\left(H H_{*}(X)_{\mathbb{Q}}\right)$ to $H H_{*}\left(\mathcal{T}^{\prime}\right) \cap H H_{*}\left(X^{\prime}\right)_{\mathbb{Q}}$. In the case $\Phi=$ Id this also proves the independence of the lattice on the embedding $\mathcal{T} \hookrightarrow D^{b}(X)$.

\subsection{Hodge-Riemann relations for matrix factorizations: homoge- neous case}

We use the rational lattices considered above to define a rational lattice in the Hochschild homology of the category $\mathrm{MF}_{\mathbb{G}_{m}}(W)$, where $W$ is a homogeneous polynomial with an isolated singularity.

Lemma 3.2.1. Let $W$ be a homogeneous polynomial with isolated singularity. Then there exists a smooth projective variety $X$ such that $\mathrm{DMF}_{\mathbb{G}_{m}}(W)$ is an admissible subcategory in $D^{b}(X)$, in a way compatible with the dg-enhancements.

Proof. Let $a=n-d$ be the Gorenstein parameter. If $a \geq 0$ then the semiorthogonal decomposition (2.21) shows that we can take $X$ to be the hypersurface $Y \subset \mathbb{P}^{n-1}$ with the equation $W=0$. In the case $a \leq 0$ we have the semiorthogonal decomposition (2.23) of $\mathrm{DMF}_{\mathbb{G}_{m}}(W)$, with $D^{b}(Y)$ as one of the pieces, where each of the remaining pieces is generated by an exceptional object. Hence, the desired $X$ can be constructed using [31, Thm. 4.15].

Combining this Lemma with Proposition 3.1.2 we equip the Hochschild homology $\mathcal{H}(W)=$ $H H_{*}\left(\mathrm{MF}_{\mathbb{G}_{m}}(W)\right)$ with a (uniquely defined) rational lattice, such that the maps on Hochschild homology induced by Fourier-Mukai transforms involving $\mathrm{MF}_{\mathbb{G}_{m}}(W)$ are compatible with this lattice. We denote by $x \mapsto \bar{x}$ the complex conjugation associated with the corresponding real structure on $\mathcal{H}(W)$.

Lemma 3.2.2. The subspace $H(W)^{\mu_{d}} \subset \mathcal{H}(W)$ coming from the decomposition (2.17) is compatible with the rational lattice in $\mathcal{H}(W)$. The projector $\Pi: \mathcal{H}(W) \rightarrow H(W)^{\mu_{d}}$ is compatible with the rational lattices.

Proof. Indeed, the operators of the $\mathbb{Z} / d$-action on $\mathcal{H}(W)$ are induced by the twist functors $E \mapsto E\{m\}$, hence they are compatible with the rational lattice in $\mathcal{H}(W)$. It remains to observe that $H(W)^{\mu_{d}}$ is the subspace of $\mathbb{Z} / d$-invariants in $\mathcal{H}(W)$, and $\Pi$ is precisely the standard projector onto it (see [36, Thm. 2.6.1(ii)]).

Let us denote by $\langle\cdot, \cdot\rangle_{W}$ the canonical pairing (2.5) on $\mathcal{H}(W)$. 
Lemma 3.2.3. For any $x \in H(W)^{\mu_{d}} \subset \mathcal{H}(W)$ and any $m \in \mathbb{Z}$ one has

$$
\left\langle x, \operatorname{ch}\left(k(m)^{\mathrm{st}}\right)\right\rangle_{W}=0 .
$$

Proof. This follows from the explicit calculation of the pairing $\langle\cdot, \cdot\rangle_{W}$ and of $\operatorname{ch}\left(k(m)^{\mathrm{st}}\right)$ in 34. Note that the $\mathbb{Z} / 2$-folding of the dg-category $\mathrm{MF}_{\mathbb{G}_{m}, d}(W)$ is naturally isomorphic to the $\mathbb{Z} / 2$-dg-category $\mathrm{MF}_{\mu_{d}}(W)$ of $\mu_{d}$-equivariant matrix factorizations (see [36, Sec. 2.1] and [34, Sec. 4.4]). The computations in [34] were done in the context of $\mathbb{Z} / 2$-dg-categories but we can use them due to the compatibility of all the Hochschild homology manipulations with the $\mathbb{Z} / 2$-folding (see remarks at the end of Sec. 2.1). By [36, Prop. 4.3.4], we see that $\operatorname{ch}\left(k^{\mathrm{st}}\right.$ ) has a trivial component in $H(W)^{\mu_{d}}$, i.e., $\Pi\left(\operatorname{ch}\left(k^{\mathrm{st}}\right)\right)=0$. It follows that $\Pi\left(\operatorname{ch}\left(k(m)^{\mathrm{st}}\right)\right)=0$ for any integer $m$ (since $\Pi$ is the projector onto the invariants of the $\mathbb{Z} / d$-action). Now the assertion follows from the fact that the summand $H(W)^{\mu_{d}}$ is orthogonal to other summands in the decomposition (2.17) with respect to $\langle\cdot, \cdot\rangle_{W}$, as the explicit formula of [34, Thm. 4.2.1] shows.

Proposition 3.2.4. Suppose we have a class $x \in H(W)^{\mu_{d}} \cap \mathcal{H}_{j}(W)$ for some $j \in \mathbb{Z}$. If $\langle x, \bar{x}\rangle_{W}=0$ then $x=0$.

Proof. Let $a$ be the Gorenstein parameter. Assume first that $a \geq 0$. Then we have a fully faithful functor $\lambda: \mathrm{DMF}_{\mathbb{G}_{m}}(W) \rightarrow D^{b}(Y)$ and the right adjoint functor $\rho$ (see (2.22) ), such that $\rho \lambda=\mathrm{Id}$. By Proposition 2.7.1, the map

$$
\rho_{*}: H^{*}(Y, \mathbb{C}) \rightarrow \mathcal{H}(W)
$$

sends classes restricted from $\mathbb{P}^{n-1}$ to the span of the Chern characters $\operatorname{ch}\left(k(m)^{\mathrm{st}}\right), m \in \mathbb{Z}$. But the latter classes are orthogonal to $H(W)^{\mu_{d}}$ with respect to $\langle\cdot, \cdot\rangle_{W}$ by Lemma 3.2.3. Hence, by adjointness of $\lambda_{*}$ and $\rho_{*}$ (see Lemma 2.4.1), $\lambda_{*}\left(H(W)^{\mu_{d}}\right)$ is left orthogonal to the image of $H^{*}\left(\mathbb{P}^{n-1}, \mathbb{C}\right) \rightarrow H^{*}(Y, \mathbb{C})$ with respect to the pairing $\langle\cdot, \cdot\rangle_{D^{b}(Y)}$. Since $\operatorname{Td}_{Y}$ is a class restricted from $\mathbb{P}^{n-1}$, using (2.6) we get that

$$
\int_{Y} \kappa\left(\lambda_{*}\left(H(W)^{\mu_{d}}\right)\right) \cdot c=0
$$

for any $c$ restricted from $\mathbb{P}^{n-1}$. Hence, for any $x \in H(W)^{\mu_{d}}, \lambda_{*}(x)$ is a primitive class. In particular, by the Lefschetz hyperplane theorem, $\lambda_{*}(x) \in H^{n-2}(Y, \mathbb{C})$. If in addition $x \in \mathcal{H}_{j}(W)$ then $\lambda_{*}(x) \in H^{p, q}(Y)$ for the unique $p, q$ such that $p+q=n-2$ and $p-q=j$. Since $\lambda_{*}$ is compatible with rational lattices, we have

$$
\lambda_{*}(\bar{x})=\tau\left(\lambda_{*}(x)\right)=(-1)^{p}(2 \pi i)^{q-p} \overline{\lambda_{*}(x)}
$$

(see (3.1)). Hence,

$$
(-1)^{p}(2 \pi i)^{q-p}\left\langle\lambda_{*}(x), \overline{\lambda_{*}(x)}\right\rangle_{D^{b}(Y)}=\left\langle\lambda_{*}(x), \lambda_{*}(\bar{x})\right\rangle_{D^{b}(Y)}=\left\langle x, \rho_{*} \lambda_{*}(\bar{x})\right\rangle_{W}=\langle x, \bar{x}\rangle_{W} .
$$

Thus, the vanishing of $\langle x, \bar{x}\rangle_{W}$ implies the vanishing of $\left\langle\lambda_{*}(x), \overline{\lambda_{*}(x)}\right\rangle_{D^{b}(Y)}$. Since $\lambda_{*}(x)$ is primitive, by Lemma 2.3.1, this implies that $\lambda_{*}(x)=0$, and so $x=0$. 
Now assume that $a \leq 0$. Then we have a fully faithful functor

$$
\rho: D^{b}(Y) \rightarrow \mathrm{DMF}_{\mathbb{G}_{m}}(W)
$$

(see (2.24) $)$, such that the image is the left orthogonal to $k^{\text {st }}, \ldots, k(a+1)^{\text {st }}$. Considering the corresponding decomposition of $\mathcal{H}(W)$ (see [23, Thm. 7.3]) we deduce that the image of $\rho_{*}$ can be identified with the left orthogonal to $\operatorname{ch}\left(k^{\mathrm{st}}\right), \ldots, \operatorname{ch}\left(k(a+1)^{\mathrm{st}}\right)$ with respect to $\langle\cdot, \cdot\rangle_{W}$. Since $H(W)^{\mu_{d}}$ lies in this left orthogonal, we deduce that any $x \in H(W)^{\mu_{d}} \cap \mathcal{H}_{j}(W)$ has form $x=\rho_{*}(y)$ for some $y \in H H_{j}(Y)$. Furthermore, by Proposition 2.7.1 and Lemma 3.2.3, we have

$$
\langle y, c\rangle_{D^{b}(Y)}=\left\langle x, \rho_{*}(c)\right\rangle_{W}=0
$$

for any $c$ restricted from $\mathbb{P}^{n-1}$. Thus, we deduce that $y$ is a primitive class. Now we can finish the proof as before, using Lemma 2.3.1 and the fact that $\rho_{*}$ is compatible with the rational lattices.

Remark 3.2.5. It is easy to see that the Chern characters $\operatorname{ch}\left(k(m)^{\mathrm{st}}\right), m \in \mathbb{Z}$, span the orthogonal complement to $H(W)^{\mu_{d}}$ in $\mathcal{H}(W)$. In the Calabi-Yau case, $d=n$, Proposition 2.7 .1 implies that the subspace $H(W)^{\mu_{d}} \subset \mathcal{H}(W)$ corresponds to the primitive part of the middle cohomology of the projective hypersurface $Y$ under the isomorphism

$$
\mathcal{H}(W) \simeq H^{*}(Y, \mathbb{C})
$$

induced by the Orlov's equivalence. Note that the images of $k(m)^{\text {st }}$ in $D^{b}(Y)$ under this equivalence are calculated explicitly in [12, Prop. 4.11].

\subsection{Hodge-Riemann relations for matrix factorizations: quasiho- mogeneous case}

Now let $W\left(x_{1}, \ldots, x_{n}\right)$ be a quasihomogeneous polynomial with an isolated singularity, such that the corresponding homogeneous polynomial $\widetilde{W}\left(y_{1}, \ldots, y_{n}\right)=W\left(y_{1}^{d_{1}}, \ldots, y_{n}^{d_{n}}\right)$ still has an isolated singularity. Let us consider the corresponding finite flat $\mathbb{G}_{m}$-equivariant morphism between affine spaces

$$
\varphi: \mathbb{A}^{n} \rightarrow \mathbb{A}^{n}:\left(y_{1}, \ldots, y_{n}\right) \mapsto\left(y_{1}^{d_{1}}, \ldots, y_{n}^{d_{n}}\right),
$$

such that $\varphi^{*} W=\widetilde{W}$. We have the corresponding functors

$$
\varphi^{*}: \operatorname{MF}_{\mathbb{G}_{m}}(W) \rightarrow \mathrm{MF}_{\mathbb{G}_{m}}(\widetilde{W}), \quad \varphi_{*}: \operatorname{MF}_{\mathbb{G}_{m}}(\widetilde{W}) \rightarrow \mathrm{MF}_{\mathbb{G}_{m}}(W),
$$

such that $\varphi_{*} \varphi^{*}(E) \simeq \varphi_{*} \mathcal{O} \otimes E$, where $\varphi_{*} \mathcal{O}$ corresponds to a free $\mathbb{C}\left[x_{1}, \ldots, x_{n}\right]$-module (with generators of various degrees).

Lemma 3.3.1. The induced maps on the Hochschild homology

$$
\varphi^{*}: \mathcal{H}(W) \rightarrow \mathcal{H}(\widetilde{W}), \quad \varphi_{*}: \mathcal{H}(\widetilde{W}) \rightarrow \mathcal{H}(W)
$$


are compatible with the decompositions (2.17), and the composition

$$
H(W)^{\mu_{d}} \stackrel{\varphi^{*}}{\longrightarrow} H(\widetilde{W})^{\mu_{d}} \stackrel{\varphi_{*}}{\longrightarrow} H(W)^{\mu_{d}}
$$

is the multiplication by $\operatorname{deg}(\varphi)=d_{1} \ldots d_{n}$.

Proof. The first assertion immediately follows from the fact that $\varphi^{*}$ and $\varphi_{*}$ commute with the twist functors $E \mapsto E\{m\}$. Next, since $\varphi_{*} \mathcal{O}$ is free of $\operatorname{rank} \operatorname{deg}(\varphi)$, we see that $\varphi_{*} \varphi^{*}(E)$ is a direct $\operatorname{sum}$ of $\operatorname{deg}(\varphi)$ twists $E\{m\}$. It remains to use the fact that these twists act trivially on the summand $H(W)^{\mu_{d}}$.

The above Lemma shows that the map

$$
\varphi^{*}: H(W)^{\mu_{d}} \rightarrow H(\widetilde{W})^{\mu_{d}}
$$

is injective and its image coincides with the image of the map $\varphi^{*} \varphi_{*}: H(\widetilde{W})^{\mu_{d}} \rightarrow H(\widetilde{W})^{\mu_{d}}$. Recall that by Lemma 3.2.2, the subspace $H(\widetilde{W})^{\mu_{d}} \subset \mathcal{H}(\widetilde{W})$ is compatible with the rational lattice in $\mathcal{H}(\widetilde{W})$. Since $\varphi^{*} \varphi_{*}$ is induced by a dg-endofunctor of $\operatorname{MF}_{\mathbb{G}_{m}}(\widetilde{W})$, it follows that the subspace

$$
\varphi^{*}\left(H(W)^{\mu_{d}}\right)=\varphi^{*} \varphi_{*}\left(H(\widetilde{W})^{\mu_{d}}\right) \subset H(\widetilde{W})^{\mu_{d}}
$$

inherits a rational lattice, so we get a rational lattice on $H(W)^{\mu_{d}}$.

Lemma 3.3.2. For any dg-functors $F: \operatorname{DMF}_{\mathbb{G}_{m}}(W) \rightarrow \mathcal{T}, G: \mathcal{T} \rightarrow \operatorname{DMF}_{\mathbb{G}_{m}}(W)$, where $\mathcal{T}$ is an admissible subcategory in $D^{b}(X)$ for some smooth and projective $X$, the induced maps

$$
H(W)^{\mu_{d}} \rightarrow \mathcal{H}(W) \stackrel{F_{*}}{\longrightarrow} H H_{*}(\mathcal{T}), \quad H H_{*}(\mathcal{T}) \stackrel{G_{*}}{\longrightarrow} \mathcal{H}(W) \stackrel{\Pi}{\longrightarrow} H(W)^{\mu_{d}}
$$

are compatible with rational structures.

Proof. By Lemma 3.3.1, to prove the assertion about $F_{*}$ we can replace it by $F_{*} \varphi_{*} \varphi^{*}$. Since $F_{*} \varphi_{*}$ is induced by a dg-functor $\operatorname{DMF}_{\mathbb{G}_{m}}(\widetilde{W}) \rightarrow \mathcal{T}$, it is compatible with the rational lattices. But the restriction of $\varphi^{*}$ to $H(W)^{\mu_{d}}$ preserves rational lattices by the definition, so the assertion follows.

To check the assertion about $\Pi G_{*}$, it is enough to prove it for the composition

$$
\varphi^{*} \Pi G_{*}=\Pi \varphi^{*} G_{*}: H H_{*}(\mathcal{T}) \rightarrow H(\widetilde{W})^{\mu_{d}} .
$$

But $\varphi^{*} G_{*}$ is induced by a dg-functor $\mathcal{T} \rightarrow \operatorname{DMF}_{\mathbb{G}_{m}}(\widetilde{W})$, so it compatible with the rational lattices. It remains to use the fact that $\Pi: \mathcal{H}(\widetilde{W}) \rightarrow H(\widetilde{W})^{\mu_{d}}$ is also compatible with the rational lattices (see Lemma 3.2.2).

Remark 3.3.3. Using the connection between $\operatorname{DMF}_{\mathbb{G}_{m}}(W)$ and the derived category of the corresponding stacky weighted projective hypersurface $\mathcal{X}$ from [29] and the recent paper [3], one can equip the Hochschild homology $\mathcal{H}(W)$ with a rational structure for any quasihomogeneous polynomial $W$ with an isolated singularity. Namely, the main result of [3] implies that $D^{b}(\mathcal{X})$ can be realized as an admissible subcategory in $D^{b}(Y)$ for $Y$ smooth and projective variety, so using the same approach as in Lemma 3.2 .1 we can realize $\operatorname{DMF}_{\mathbb{G}_{m}}(W)$ as such a subcategory. 
As before, we denote by $x \mapsto \bar{x}$ the conjugation associated with the real structure on $H(W)^{\mu_{d}}$.

Proposition 3.3.4. Let $W$ be a quasihomogeneous polynomial with an isolated singularity such that $\widetilde{W}$ still has an isolated singularity. Given a class $x \in H(W)^{\mu_{d}} \cap \mathcal{H}_{j}(W)$ for some $j \in \mathbb{Z}$, if $\langle x, \bar{x}\rangle_{W}=0$ then $x=0$.

Proof. The map $\varphi^{*}: H(W)^{\mu_{d}} \rightarrow H(\widetilde{W})^{\mu_{d}}$ is rational, so using adjointness of $\left(\varphi^{*}, \varphi_{*}\right)$ and Lemma 2.4.1 we get

$$
\left\langle\varphi^{*} x, \overline{\varphi^{*}(x)}\right\rangle_{\widetilde{W}}=\left\langle\varphi^{*} x, \varphi^{*}(\bar{x})\right\rangle_{\widetilde{W}}=\left\langle x, \varphi_{*} \varphi^{*}(\bar{x})\right\rangle_{W}=\operatorname{deg}(\varphi)\langle x, \bar{x}\rangle_{W}=0 .
$$

Also we have $\varphi^{*} x \in H(\widetilde{W})^{\mu_{d}} \cap \mathcal{H}_{j}(\widetilde{W})$. Hence, by Proposition 3.2.4, we obtain $\varphi^{*} x=0$, and so by Lemma 3.3.1, $x=0$.

Remark 3.3.5. The idea of using the relation between matrix factorizations of $W$ and $\widetilde{W}$ to deduce results about $\mathcal{H}(W)$ was inspired by a similar method in [27, Sec. 6].

\section{Homogeneity}

\subsection{Calculations with Koszul matrix factorizations}

Let $A$ and $B$ be $\mathbb{G}_{m}$-vector bundles over a scheme $S$ (where $\mathbb{G}_{m}$ acts trivially on $S$ ), $\alpha \in$ $H^{0}\left(\operatorname{tot}(A), p^{*} B^{\vee}\{d\}\right)$ and $\beta \in H^{0}\left(\operatorname{tot}(A), p^{*} B\right)$ be $\mathbb{G}_{m}$-invariant sections, where $p: \operatorname{tot}(A) \rightarrow$ $S$ is the projection. Assume that $\alpha$ and $\beta$ are orthogonal and have common zeros only on the zero section in $\operatorname{tot}(A)$. Let

$$
E=\{\alpha, \beta\} \in \mathrm{MF}_{\mathbb{G}_{m}, d}(\operatorname{tot}(A), 0)
$$

be the corresponding Koszul matrix factorization of 0 on $\operatorname{tot}(A)$ (see Section 2.5). The following homogeneity property follows from the results of [33] and [10] (it also appears implicitly in the proof of [36, Prop. 5.6.1]).

Proposition 4.1.1. In the above situation we have

$$
\operatorname{Td}(B) \operatorname{Td}(A)^{-1} \operatorname{ch}\left(\overline{\operatorname{com}}\left(p_{*} E\right)\right) \in H^{2(\operatorname{rk} B-\operatorname{rk} A)}(S) .
$$

Proof. Note that

$$
\operatorname{ch}\left(\overline{\operatorname{com}}\left(p_{*} E\right)\right)=\operatorname{ch}\left(p_{*} E\right):=\operatorname{ch}\left(H^{\text {even }}\left(p_{*} E\right)\right)-\operatorname{ch}\left(H^{\text {odd }}\left(p_{*} E\right)\right),
$$

where on the right we view $p_{*} E$ as a $\mathbb{Z} / 2$-graded complex. It is enough to show that in the Chow group $A^{*}(S)_{\mathbb{Q}}$ one has

$$
\operatorname{Td}(B) \operatorname{Td}(A)^{-1} \operatorname{ch}\left(p_{*} E\right) \in A^{\mathrm{rk} B-\mathrm{rk} A}(S)_{\mathbb{Q}} .
$$


By [10, Lemma 5.3.8], one has

$$
\operatorname{Td}(A)^{-1} \operatorname{ch}\left(p_{*} E\right)=\operatorname{ch}_{S}^{\text {tot }} A(E) \cdot[p]
$$

where $\operatorname{ch}_{S}^{\text {tot } A}(E) \in A^{*}(S \rightarrow \operatorname{tot}(A))$ is the localized Chern character of the $\mathbb{Z} / 2$-graded complex $E$ in the relative Chow group for the zero section embedding $S \rightarrow \operatorname{tot}(A)$ (see [33, Sec. 2.2]), and $[p] \in A^{-r k A}(\operatorname{tot} A \rightarrow S)$ is the orientation class of $p$. Now by [33, Thm. 3.2], the class

$$
\operatorname{Td}(B) \operatorname{ch}_{S}^{\operatorname{tot} A}(E) \in A^{*}(S \rightarrow \operatorname{tot} A)
$$

is concentrated in degree $\operatorname{rk} B$. To get the class we need, one has to multiply the above class with the orientation class $[p]$ that lives in degree $-r k A$, hence the result.

Now let us consider the setup of Sec. 1.2, Note that the functor $\Phi: \operatorname{DMF}_{\mathbb{G}_{m}}(W) \rightarrow D^{b}(X)$ in (1.5) is of the form $\Phi=\Phi_{P}($ see $(2.16)$ ) for the diagram

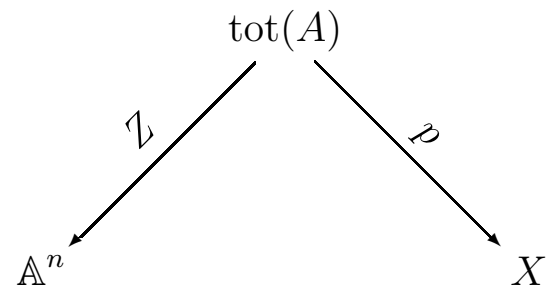

and $P=\{\alpha, \beta\}$. Note also that in this case $\operatorname{DMF}_{\mathbb{G}_{m}}(W)=\overline{\mathrm{DMF}}_{\mathbb{G}_{m}}(W)$ is the usual homotopy category of matrix factorizations.

We start by computing the left adjoint functor $\Psi: D^{b}(X) \rightarrow \operatorname{DMF}_{\mathbb{G}_{m}}(W)$ to $\Phi$.

Lemma 4.1.2. In the notation of (2.16), one has $\Psi=\Psi_{P^{\prime}}$, where

$$
P^{\prime}=\left\{\alpha^{\prime}, \beta^{\prime}\right\} \otimes p^{*}\left(\operatorname{det}^{-1}(A) \otimes \omega_{X}\right)[N],
$$

with $\alpha^{\prime}=\beta \in p^{*} B, \beta^{\prime}=-\alpha \in p^{*} B^{\vee}\{d\}, N=\operatorname{dim} X+\operatorname{rk} A-n$. Thus, the Koszul matrix factorization $\left\{\alpha^{\prime}, \beta^{\prime}\right\}$ is of the same type as $\{\alpha, \beta\}$ but with $B$ being replaced by $B^{\vee}\{d\}$.

Proof. By Proposition 2.6.2, we have $\Psi=\Psi_{P^{\prime}}$ with

$$
P^{\prime}=P^{\vee} \otimes \omega_{Z}[N]
$$

Recall that the dual matrix factorization $P^{\vee}$ has the even part $P_{0}^{\vee}$, the odd part $P_{1}^{\vee}\{-d\}$, and the differential $\delta_{P \vee}$ determined by the rule

$$
\left\langle\delta_{P^{\vee}}(\xi), x\right\rangle=(-1)^{\operatorname{deg}(\xi)}\left\langle\xi, \delta_{P}(x)\right\rangle .
$$

Thus, disregarding the $\mathbb{G}_{m}$-action we can identify $P^{\vee}$ with $\bigwedge^{*}\left(p^{*} B\right)$ using the isomorphism

$$
\bigwedge^{*}\left(p^{*} B\right) \stackrel{\sim}{\longrightarrow}\left(\bigwedge^{*}\left(p^{*} B^{\vee}\right)\right)^{\vee}: b_{1} \wedge \ldots \wedge b_{p} \mapsto\left[w \mapsto\left(\iota_{b_{1}} \circ \ldots \circ \iota_{b_{p}}\right)(w)_{0}\right]
$$


where the subscript 0 means taking the component in $\Lambda^{0}$. Under this identification, with the above sign convention, the operator dual to $\alpha \wedge$ is $-\iota_{\alpha}$, and the operator dual to $\iota_{\beta}$ is $\beta \wedge$. The $\mathbb{G}_{m}$-weights work out so that we have an identification

$$
P^{\vee} \simeq\left\{\alpha^{\prime}, \beta^{\prime}\right\}
$$

Taking into account the isomorphism

$$
\omega_{Z} \simeq \omega_{\mathrm{tot} A} \simeq \omega_{p} \otimes p^{*} \omega_{X} \simeq p^{*}\left(\operatorname{det}^{-1}(A) \otimes \omega_{X}\right)
$$

we get the assertion.

Lemma 4.1.3. In the above situation the composed functor $\Phi \circ \Psi: D^{b}(X) \rightarrow D^{b}(X)$ is associated with a kernel $K \in D^{b}(X \times X)$ such that $[K]=d \cdot\left[K^{\prime}\right]$ in $K_{0}(X \times X)$, where

$$
K^{\prime}=\overline{\operatorname{com}}\left(p_{*}^{(2)}\left\{\alpha^{(2)}, \beta^{(2)}\right\}\right) \otimes p_{1}^{*}\left(\operatorname{det}^{-1}(A) \otimes \omega_{X}\right)[N]
$$

for a Koszul matrix factorization of zero $\left\{\alpha^{(2)}, \beta^{(2)}\right\}$ on the vector bundle

$$
p^{(2)}: \operatorname{tot}(A) \times_{\mathbb{A}^{n}} \operatorname{tot}(A) \rightarrow X \times X
$$

associated with the sections $\alpha^{(2)}=\left(\alpha, \alpha^{\prime}\right) \in p_{1}^{*} B^{\vee}\{d\} \oplus p_{2}^{*} B, \beta^{(2)}=\left(\beta, \beta^{\prime}\right) \in p_{1}^{*} B \oplus p_{2}^{*} B^{\vee}\{d\}$. Furthermore, $\alpha^{(2)}$ and $\beta^{(2)}$ have common zeros only on the zero section of the bundle $p^{(2)}$.

Proof. The computation of the composition follows from Lemma 2.6.1. The fact about the common zeros of $\alpha^{(2)}$ and $\beta^{(2)}$ follows from the similar fact about $(\alpha, \beta)$ and $\left(\alpha^{\prime}, \beta^{\prime}\right)$.

Note that the bundle $\operatorname{tot}(A) \times_{\mathbb{A}^{n}} \operatorname{tot}(A)$ over $X \times X$ has $\operatorname{rank} 2 \operatorname{rk} A-n$. Thus, by Proposition 4.1.1, we have

$\operatorname{Td}\left(p_{1}^{*} B^{\vee}\right) \operatorname{Td}\left(p_{2}^{*} B\right) \operatorname{Td}\left(p_{1}^{*} A\right)^{-1} \operatorname{Td}\left(p_{2}^{*} A\right)^{-1} \operatorname{ch}\left(K \otimes p_{1}^{*}\left(\operatorname{det}(A) \otimes \omega_{X}^{-1}\right)\right) \in H^{2(2 \operatorname{rk} B-2 \operatorname{rk} A+n)}(X \times X)$.

Using the formula

$$
\operatorname{Td}\left(E^{\vee}\right)=\operatorname{Td}(E) \cdot \operatorname{ch}(\operatorname{det}(E))^{-1},
$$

we can rewrite the above class as

$$
p_{1}^{*}\left(\operatorname{ch}\left(\omega_{X}\right)^{-1} \operatorname{Td}\left(B^{\vee}\right) \operatorname{Td}\left(A^{\vee}\right)^{-1}\right) \cdot p_{2}^{*}\left(\operatorname{Td}(B) \operatorname{Td}(A)^{-1}\right) \operatorname{ch}(K) \in H^{2 D}(X \times X),
$$

where $D$ is given by (1.6).

\subsection{The proof of Theorem 1.2 .1}

We start by reformulating the statement using the canonical bilinear forms on Hochschild homology. Let $\phi=\Phi_{*}: \mathcal{H}(W)=H H_{*}\left(\mathrm{MF}_{\mathbb{G}_{m}}(W)\right) \rightarrow H^{*}(X)$ be the map induced by $\Phi=\Phi_{P}$ on Hochschild homology. Let us set

$$
\alpha=\operatorname{Td}(B) \operatorname{Td}(A)^{-1}
$$


By the nondegeneracy of the Poincaré pairing, and by (2.6), the left orthogonal to $H^{i}(X) \subset H^{*}(X)$ with respect to $\langle\cdot, \cdot\rangle_{D^{b}(X)}$ is the subspace

$$
{ }^{\perp} H^{i}(X)=\bigoplus_{j \neq 2 \operatorname{dim} X-i} \kappa\left(\operatorname{Td}_{X}\right)^{-1} \cdot H^{j}(X) \subset H^{*}(X)
$$

where $\kappa$ is given by (2.8). Since $\left\langle x, \alpha^{-1} \cdot y\right\rangle_{D^{b}(X)}=\left\langle\kappa\left(\alpha^{-1}\right) \cdot x, y\right\rangle_{D^{b}(X)}$, we deduce that

$$
{ }^{\perp}\left(\alpha^{-1} \cdot H^{i}(X)\right)=\bigoplus_{j \neq 2 \operatorname{dim} X-i} \kappa\left(\alpha \cdot \mathrm{Td}_{X}\right)^{-1} \cdot H^{j}(X) \subset H^{*}(X)
$$

(note that $\kappa(\alpha)$ and $\kappa\left(\operatorname{Td}_{X}\right)$ live in even degrees, so they commute with any cohomology class).

By the nondegeneracy of $\langle\cdot, \cdot\rangle_{D^{b}(X)}$, to show that $\phi(x) \in \alpha^{-1} \cdot H^{D}(X)$ for all $x \in \mathcal{H}(W)$, it is enough to prove that

$$
\psi\left({ }^{\perp}\left(\alpha^{-1} \cdot H^{D}(X)\right)\right)=0,
$$

where $\psi$ is the left adjoint operator to $\phi$. Note that by Lemma 2.4.1, we have $\psi=\Psi_{*}$. Thus, taking into account (4.2), we should check that for each $j \neq 2 \operatorname{dim} X-D$ one has

$$
\psi\left(\kappa\left(\alpha \cdot \operatorname{Td}_{X}^{-1}\right) \cdot H^{j}(X)\right)=0
$$

We are going to use the rational lattices on the relevant Hochschild homology introduced in Sec. 3. We denote by $x \mapsto \bar{x}$ (resp., $\tau$ ) the corresponding operation of complex conjugation on $\mathcal{H}(W)$ (resp., $\left.H^{*}(X, \mathbb{C})\right)$.

Let $y \in H^{p, q}(X) \subset H^{j}(X)$, and set $y^{\prime}=\kappa\left(\alpha \operatorname{Td}_{X}^{-1}\right) \cdot y$. Note that $y^{\prime}$, viewed as an element of Hochschild homology, lives in the single degree $p-q$. Hence, the same is true about $z=\psi\left(y^{\prime}\right)$. Also, since by Lemma 2.6.3, $\phi \Pi=\phi$, it follows that $\Pi \psi=\psi$, so $z \in H(W)^{\mu_{d}}$. By Proposition 3.3.4, $z=0$ if and only if $\langle\bar{z}, z\rangle_{W}=0$. Thus, it is enough to prove that

$$
\left\langle\overline{\psi\left(y^{\prime}\right)}, \psi\left(y^{\prime}\right)\right\rangle_{W}=0
$$

provided $j \neq 2 d-D$.

We will use the following fact about the involution $\kappa$. For any vector bundle $V$ on $X$ one has

$$
\kappa(\operatorname{Td}(V))=\operatorname{Td}\left(V^{\vee}\right)=\operatorname{Td}(V) \cdot \operatorname{ch}(\operatorname{det}(V))^{-1} .
$$

In particular, the classes $\kappa(\operatorname{Td}(V))$ and $\kappa\left(\operatorname{Td}(V)^{-1}\right)=\kappa(\operatorname{Td}(V))^{-1}$ belong to the rational lattice $J\left(H^{*}(X, \mathbb{Q})\right)$ (see Section 3.1).

Thus, the class $\kappa\left(\alpha \operatorname{Td}_{X}^{-1}\right)$ is in $J\left(H^{*}(X, \mathbb{Q})\right)$. Hence, using (3.1) we get

$$
\tau\left(y^{\prime}\right)=\kappa\left(\alpha \operatorname{Td}_{X}^{-1}\right) \cdot \tau(y)=(-1)^{p}(2 \pi i)^{q-p} \kappa\left(\alpha \operatorname{Td}_{X}^{-1}\right) \cdot \bar{y}
$$

Since the operator $\psi=\Pi \psi$ is compatible with the rational lattices (see Lemma 3.3.2), we deduce

$$
\overline{\psi\left(y^{\prime}\right)}=\psi\left(\tau\left(y^{\prime}\right)\right)=(-1)^{p}(2 \pi i)^{q-p} \psi\left(\kappa\left(\alpha \operatorname{Td}_{X}^{-1}\right) \cdot \bar{y}\right)
$$


Hence, by adjointness of the pair $(\psi, \phi)$,

$$
\left\langle\overline{\psi\left(y^{\prime}\right)}, \psi\left(y^{\prime}\right)\right\rangle_{W}=(-1)^{p}(2 \pi i)^{q-p}\left\langle\kappa\left(\alpha \mathrm{Td}_{X}^{-1}\right) \cdot \bar{y}, \phi \psi\left(y^{\prime}\right)\right\rangle_{D^{b}(X)}
$$

Recall that $\phi \psi=\Phi_{*} \Psi_{*}=(\Phi \circ \Psi)_{*}$ is induced by the Fourier-Mukai functor with the kernel $K$, as in Lemma 4.1.3. Thus, by Lemma 2.4.2, we have

$$
\phi \psi\left(y^{\prime}\right)=\operatorname{tr}_{12}\left(y^{\prime} \otimes \operatorname{ch}(K)\right)=\int_{p_{2}} p_{1}^{*}\left(\operatorname{Td}_{X} \cdot y^{\prime}\right) \operatorname{ch}(K)
$$

where

$$
\int_{p_{2}}:=\left(\int_{X} \otimes \mathrm{id}\right): H^{*}(X \times X) \simeq H^{*}(X) \otimes H^{*}(X) \rightarrow H^{*}(X) .
$$

Taking into account the relation (4.3) we get

$$
\phi \psi\left(y^{\prime}\right)=\int_{p_{2}} p_{1}^{*}\left(\operatorname{ch}\left(\omega_{X}\right)^{-1} \kappa(\alpha) \cdot y\right) \operatorname{ch}(K) .
$$

Thus, we can rewrite (4.4) as follows:

$$
\begin{aligned}
& (-1)^{p}(2 \pi i)^{p-q}\left\langle\overline{\psi\left(y^{\prime}\right)}, \psi\left(y^{\prime}\right)\right\rangle_{W}=\left\langle\kappa\left(\alpha \operatorname{Td}_{X}^{-1}\right) \cdot \bar{y}, \phi \psi\left(y^{\prime}\right)\right\rangle_{D^{b}(X)}=\int_{X} \alpha \cdot \kappa(\bar{y}) \cdot \phi \psi\left(y^{\prime}\right)= \\
& (-1)^{p} \int_{X} \bar{y} \cdot\left(\alpha \cdot \int_{p_{2}} p_{1}^{*}\left(\operatorname{ch}\left(\omega_{X}\right)^{-1} \kappa(\alpha) y\right) \cdot \operatorname{ch}(K)\right)= \\
& (-1)^{p} \int_{X \times X} p_{2}^{*}(\bar{y}) \cdot p_{1}^{*}(y) \cdot\left(p_{1}^{*}\left(\operatorname{ch}\left(\omega_{X}\right)^{-1} \kappa(\alpha)\right) \cdot p_{2}^{*} \alpha \cdot \operatorname{ch}(K)\right) .
\end{aligned}
$$

Note that the condition (4.1) simply means that

$$
p_{1}^{*}\left(\operatorname{ch}\left(\omega_{X}\right)^{-1} \kappa(\alpha)\right) \cdot p_{2}^{*} \alpha \cdot \operatorname{ch}(K) \in H^{2 D}(X \times X) .
$$

On the other hand, $p_{2}^{*}(\bar{y}) \cdot p_{1}^{*}(y) \in H^{2 j}(X \times X)$. Thus, the above integral vanishes unless $2 j+2 D=4 \operatorname{dim} X$. In other words, it is zero unless $j=2 \operatorname{dim} X-D$.

\subsection{Proof of Theorem 1.1 .1}

Let $W$ be a quasihomogeneous polynomial of degree $d$ as in Theorem [1.1.1, and let $G$ be a finite group of diagonal symmetries of $W$, containing the exponential grading operator $J$. Recall that for $\gamma_{1}, \ldots, \gamma_{r} \in G$ the maps

$$
\phi_{g}\left(\gamma_{1}, \ldots, \gamma_{r}\right): H\left(W_{\gamma_{1}}\right)^{G} \otimes \ldots \otimes H\left(W_{\gamma_{r}}\right)^{G} \rightarrow H^{*}(\mathcal{S}, \mathbb{C}),
$$

where $\mathcal{S}=\mathcal{S}_{g, \mu_{d}}\left(\gamma_{1}, \ldots, \gamma_{r}\right)$ is the moduli of $\Gamma$-spin structures associated with $G$, giving the algebraic FJRW cohomological field theory, are obtained in the following way (see [36, Sec. 5.1]). First, we consider the potential $W=W_{\gamma_{1}} \oplus \ldots \oplus W_{\gamma_{r}}$, and the map

$$
\phi: H H_{*}\left(\mathrm{MF}_{\mathbb{G}_{m}}(W)\right) \rightarrow H^{*}(\mathcal{S}, \mathbb{C})
$$


defined as in Sec. 1.2, using a certain $\mathbb{G}_{m}$-equivariant Koszul matrix factorization $\{\alpha, \beta\}$ of $-Z^{*} W$ on the total space of a vector bundle $p: \operatorname{tot}(A) \rightarrow \mathcal{S}$, equipped with a map $Z: \operatorname{tot}(A) \rightarrow \mathbb{A}^{n}$. The definition of the Koszul matrix factorization $\{\alpha, \beta\}$ is rather involved (see [36, Sec. 4]) and will not be repeated here: for our purposes we only need to know that it is supported on the zero section in $\operatorname{tot}(A)$. One difference from the framework of Sec. 1.2 is that $\mathcal{S}$ is not a variety, but a DM-stack. However, there is still a natural map

$$
H H_{*}(\mathcal{S}) \rightarrow H^{*}(\mathcal{S}, \mathbb{C})
$$

(see [36, Eq. (5.6)]), which we use to define $\phi$ with values in $H^{*}(\mathcal{S}, \mathbb{C})$. Now the map $\phi_{g}$ is obtained by restricting $\operatorname{Td}(A)^{-1} \operatorname{Td}(B) \phi$ to the subspace

$$
H\left(W_{\gamma_{1}}\right)^{\mu_{d}} \otimes \ldots \otimes H\left(W_{\gamma_{r}}\right)^{\mu_{d}} \subset H(W)^{\mu_{d}} .
$$

(here we use the fact that $\operatorname{Td}(A) \operatorname{Td}(B)^{-1}=\operatorname{Td}\left(R \pi_{*}\left(\bigoplus_{j=1}^{n} \mathcal{L}_{j}\right)\right.$ ), where $\left(\mathcal{L}_{\bullet}\right)$ comes from a universal generalized spin-structure on $\mathcal{S})$.

Actually, in [36] we consider a bigger group $\Gamma$ and a $\Gamma$-equivariant matrix factorization $\mathbf{P}=(p, Z)_{*}\{\alpha, \beta\}$ of $-W$ on $\mathbb{A}^{n} \times X$ to produce a map of $\mathbb{C}\left[G^{*}\right]$-modules

$$
H H_{*}\left(\mathrm{MF}_{\Gamma}(W)\right) \rightarrow H^{*}(\mathcal{S}, \mathbb{C}) \otimes \mathbb{C}\left[G^{*}\right],
$$

where $G^{*}$ is the dual group to $G$. To get $\phi_{g}\left(\gamma_{1}, \ldots, \gamma_{r}\right)$ we specialize this map using the evaluation at 1 homomorphism $\mathbb{C}\left[G^{*}\right] \rightarrow \mathbb{C}$, compose the resulting map with a natural embedding

$$
H\left(W_{\gamma_{1}}\right)^{G} \otimes \ldots \otimes H\left(W_{\gamma_{r}}\right)^{G} \rightarrow H H_{*}\left(\operatorname{MF}_{\Gamma}(W)\right) \otimes_{\mathbb{C}\left[G^{*}\right]} \mathbb{C},
$$

and twist by $\operatorname{Td}\left(R \pi_{*}\left(\bigoplus_{j=1}^{n} \mathcal{L}_{j}\right)\right)^{-1}$. It is easy to check that one gets the same map by passing to $\mathbb{G}_{m}$-equivariant matrix factorizations and then applying the above procedure.

The map (4.5) is defined using a finite flat surjective morphism $\pi: X \rightarrow \mathcal{S}$, where $X$ is a smooth projective variety (the existence of such maps is a general fact about smooth proper DM-stacks over $\mathbb{C}$ with projective coarse moduli spaces - see [21, Thm. 4.4], [22, Thm. 2.1]). In fact, (4.5) factors through the pull-back map

$$
\pi_{*}: H H_{*}(\mathcal{S}) \rightarrow H H_{*}(X) \simeq H^{*}(X, \mathbb{C}),
$$

followed by the degree preserving map $H^{*}(X, \mathbb{C}) \rightarrow H^{*}(\mathcal{S}, \mathbb{C})$. Thus, it is enough to prove the required purity of dimension over $X$. Taking into account the equality

$$
D_{g}\left(\gamma_{1}, \ldots, \gamma_{r}\right)=-\operatorname{rk} R \pi_{*}\left(\bigoplus_{j=1}^{n} \mathcal{L}_{j}\right)=\operatorname{rk} B-\operatorname{rk} A,
$$

we see that Theorem 1.2.1 would imply the dimension property (1.2) provided we check that the polynomial $W_{\gamma_{1}} \oplus \ldots \oplus W_{\gamma_{r}}$ satisfies the assumptions of that Theorem, i.e., each homogeneous polynomial $\widetilde{W_{\gamma_{i}}}$ still has an isolated singularity at 0 . But this follows easily from Lemma 4.3 .1 below. 
Lemma 4.3.1. Let $W\left(x_{1}, \ldots, x_{n}\right)$ be a quasihomogeneous polynomial with an isolated singularity, where $\operatorname{deg}\left(x_{i}\right)=d_{i}>0$. Let $I \subset[1, n]$ be the set of $i$ such that $d_{i}>1$. Then $\widetilde{W}\left(y_{1}, \ldots, y_{n}\right)=W\left(y_{1}^{d_{1}}, \ldots, y_{n}^{d_{n}}\right)$ still has an isolated singularity if and only for every subset $J \subset I$ the restriction $\left.W\right|_{\mathbb{A}_{J}^{n}}$ has an isolated singularity, where $\mathbb{A}_{J}^{n} \subset \mathbb{A}^{n}$ is the linear subspace given by $x_{j}=0$ for all $j \in J$.

Proof. We have $\partial_{y_{i}} \widetilde{W}(y)=d_{i} y_{i}^{d_{i}-1} \partial_{x_{i}} W(\varphi(y))$, where $\varphi\left(y_{1}, \ldots, y_{n}\right)=\left(y_{1}^{d_{1}}, \ldots, y_{n}^{d_{n}}\right)$. Thus, for $i \notin I$ we have $\partial_{y_{i}} \widetilde{W}(y)=0$ if and only if $\partial_{x_{i}} W(\varphi(y))=0$. On the other hand, for $i \in I$ we have $\partial_{y_{i}} \widetilde{W}(y)=0$ if and only if either $y_{i}=0$ or $\partial_{x_{i}} W(\varphi(y))=0$. This easily implies that $y$ is a critical point of $\widetilde{W}$ if and only if $\varphi(x)$ is a critical point of $\left.W\right|_{\mathbb{A}_{J}^{n}}$ for some subset $J \subset I$.

\section{APPENDIX: A compatibility involving the Grothendieck duality.}

Let $f: Y \rightarrow Z$ be a separated morphism of finite type between Noetherian schemes. We denote by $f^{+}: D^{+}(q \operatorname{coh}(Y)) \rightarrow D^{+}(q \operatorname{coh}(Z))$ the extraordinary inverse image functor (see [18] where it is denoted by $\left.f^{!}\right)$. Then for any $F \in D^{b}(Y)$ such that the support of $F$ is proper over $Z$, we have a canonical morphism

$$
c_{f, F}: F \rightarrow f^{+} f_{*} F .
$$

Indeed, this can be reduced to a similar map in the case when $f$ is proper: let $F=i_{*} F^{\prime}$, where $i: Y^{\prime} \rightarrow Y$ is a closed subscheme, proper over $Z$, and $F^{\prime} \in D^{b}\left(Y^{\prime}\right)$. Then we have $f_{*} F \simeq f_{*}^{\prime} F^{\prime}$, where $f^{\prime}=f \circ i$. Since $f^{\prime}$ is proper, we have a canonical map

$$
c_{f^{\prime}, F^{\prime}}: F^{\prime} \rightarrow\left(f^{\prime}\right)^{+} f_{*}^{\prime} F^{\prime} \simeq i^{+} f^{+} f_{*} F .
$$

By adjunction of $i_{*}$ and $i^{+}$we get the required map

$$
F=i_{*} F^{\prime} \rightarrow f^{+} f_{*} F
$$

Furthermore, for such $F$ and for any $G \in D^{b}(Z)$ the natural map

$$
\operatorname{Hom}\left(f_{*} F, G\right) \rightarrow \operatorname{Hom}\left(f^{+} f_{*} F, f^{+} G\right) \rightarrow \operatorname{Hom}\left(F, f^{+} G\right),
$$

where the second arrow is induced by $c_{F}$, is an isomorphism (again this easily reduces to the case when $f$ is proper).

The fact that $c_{f, F}$ does not depend on a choice of the subscheme $Y^{\prime}$ follows from the compatibility of the maps $c_{f, F}$ with compositions (for proper maps). Namely, for a morphism $g: Z \rightarrow T$ we have a commutative triangle

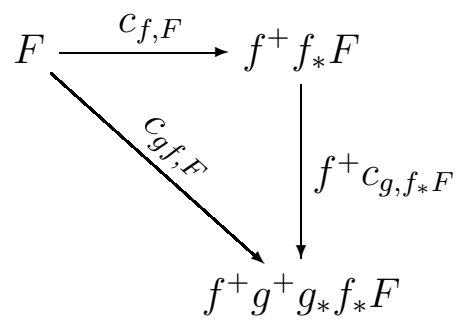


The above picture extends to matrix factorizations. Namely, let us assume that we have the following situation:

$(\star): Y$ and $Z$ are smooth $\mathbb{G}_{m}$-varieties admitting $\mathbb{G}_{m}$-equivariant ample line bundles; $f: Y \rightarrow Z$ is a smooth $\mathbb{G}_{m}$-equivariant morphism; $W$ is a function on $Z$ of weight $d>0$ with respect to the $\mathbb{G}_{m}$-action, which is not a zero divisor.

Then canonical morphism (5.1) can be constructed for $F \in \operatorname{MF}_{\mathbb{G}_{m}}\left(Y, f^{*} W\right)$, with proper support over $Z$, using Corollary 2.5.7, and the compatibility (5.3) still holds provided $g$ is also smooth $\mathbb{G}_{m}$-equivariant and $W=g^{*} W^{\prime}$.

Next, let us consider the fibered product $Y \times_{Z} Y$ with its two projections $p_{1}, p_{2}: Y \times_{Z} Y \rightarrow$ $Y$, and let $\delta: Y \rightarrow Y \times{ }_{Z} Y$ be the diagonal embedding. Let us also set

$$
\pi=f \circ p_{1}=f \circ p_{2}: Y \times_{Z} Y \rightarrow Z .
$$

Then for any $F \in D^{b}(Y)$ we have a canonical morphism

$$
\alpha_{F}: \delta_{*} F \rightarrow p_{2}^{+} F
$$

on $Y \times_{Z} Y$, which corresponds by adjunction to the identity map

$$
F \rightarrow \delta^{+} p_{2}^{+} F \simeq F .
$$

Equivalently, it corresponds by adjunction to the identity map

$$
F \simeq p_{2 *} \delta_{*} F \rightarrow F
$$

(note that $\delta_{*} F$ is supported on the diagonal which is proper over $Y$ ). Exchanging the roles of the factors in $Y \times_{Z} Y$ we get canonical morphisms

$$
\alpha_{F}^{\prime}: \delta_{*} F \rightarrow p_{1}^{+} F .
$$

As before, we can define similar morphisms for $F \in \operatorname{MF}_{\mathbb{G}_{m}}\left(Y, f^{*} W\right)$ assuming the situation $(\star)$.

We will need the following properties of the maps $\alpha$ and $\alpha^{\prime}$. We set $\mathcal{D}_{f}=f^{+} \mathcal{O}_{Z} \simeq$ $\omega_{f}[\operatorname{dim} Y-\operatorname{dim} Z]$.

Lemma 5.0.2. (i) In the above situation, assuming that $F \in D^{b}(Y)$ has the support that is proper over $Z$, we have a commutative triangle

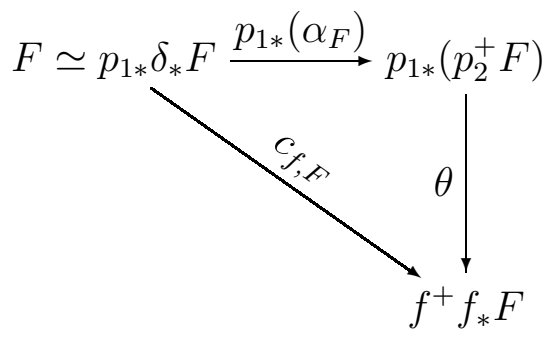

where $\theta$ is the base change map, which corresponds via the isomorphism (5.2) for $p_{1}$ to the map

$$
p_{2}^{+} F \stackrel{p_{2}^{+} c_{f, F}}{\longrightarrow} p_{2}^{+} f^{+} f_{*} F \simeq p_{1}^{+} f^{+} f_{*} F
$$


(note that the map from the support of $p_{2}^{+} F$ to $Y$, induced by $p_{1}$, is proper). The similar assertion holds for $F \in \mathrm{MF}_{\mathbb{G}_{m}}\left(Y, f^{*} W\right)$, with proper support over $Z$, assuming the situation $(\star)$.

(ii) For $F, G \in D^{b}(Y)$, or, assuming the situation $(\star)$, for $F \in \operatorname{Per}_{\mathbb{G}_{m}}(Y), G \in \mathrm{MF}_{\mathbb{G}_{m}}\left(Y, f^{*} W\right)$, the diagram

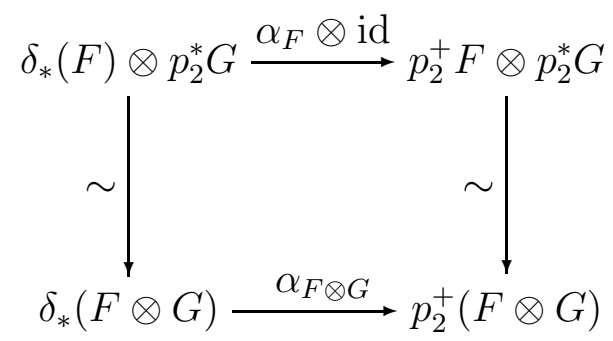

is commutative. The same property holds for the maps $\alpha^{\prime}$.

(iii) Under the natural identification $p_{1}^{+} \mathcal{D}_{f} \simeq p_{2}^{+} \mathcal{D}_{f} \simeq p_{1}^{*} \mathcal{D}_{f} \otimes p_{2}^{*} \mathcal{D}_{f}$ one has

$$
\alpha_{\mathcal{D}_{f}}=\alpha_{\mathcal{D}_{f}}^{\prime} \in \operatorname{Hom}\left(\delta_{*} \mathcal{D}_{f}, p_{1}^{*} \mathcal{D}_{f} \otimes p_{2}^{*} \mathcal{D}_{f}\right) .
$$

Proof. (i) Applying the compatibility (5.3) to the maps $f, p_{1}$ and the object $\delta_{*} F$ we get that $c_{\pi, \delta_{*} F}=c_{f p_{1}, \delta_{*} F}$ is equal to the composition

$$
\delta_{*} F \rightarrow p_{1}^{+} p_{1 *} \delta_{*} F=p_{1}^{+} F \stackrel{p_{1}^{+} c_{f, F}}{\longrightarrow} p_{1}^{+} f^{+} f_{*} F .
$$

In other words, the map $c_{f, F}$ corresponds to $c_{\pi, \delta_{*} F}$ under the adjunction isomorphism (5.2). Thus, we have to show that $c_{\pi, \delta_{*} F}=c_{f p_{2}, \delta_{*} F}$ is equal to the composition

$$
\delta_{*} F \stackrel{\alpha_{F}}{\longrightarrow} p_{2}^{+} F \stackrel{p_{2}^{+} c_{f, F}}{\longrightarrow} p_{2}^{+} f^{+} f_{*} F \simeq p_{1}^{+} f^{+} f_{*} F .
$$

But this immediately follows from the compatibility (5.3) applied to the maps $f, p_{2}$ and the object $\delta_{*} F$.

(ii) First, let us consider the case of sheaves. By reversing the direction of the isomorphism of the left vertical arrow and using the adjointness of $\left(\delta_{*}, \delta^{+}\right)$we reformulate the required commutativity as showing that the following composition is the identity map:

$F \otimes G \rightarrow \delta^{+} \delta_{*}(F \otimes G) \stackrel{\sim}{\longrightarrow} \delta^{+}\left(\delta_{*}(F) \otimes p_{2}^{*} G\right) \stackrel{\alpha_{F}}{\longrightarrow} \delta^{+}\left(p_{2}^{+} F \otimes p_{2}^{*} G\right) \stackrel{\sim}{\longrightarrow} \delta^{+} p_{2}^{+}(F \otimes G) \simeq F \otimes G$,

where the first arrow is the adjunction map. Now we use the following standard compatibility of the canonical morphisms $t_{f, A, B}: f^{+}(A) \otimes f^{*}(B) \rightarrow f^{+}(A \otimes B)$ with the projection formula: for a proper map $f$ the square

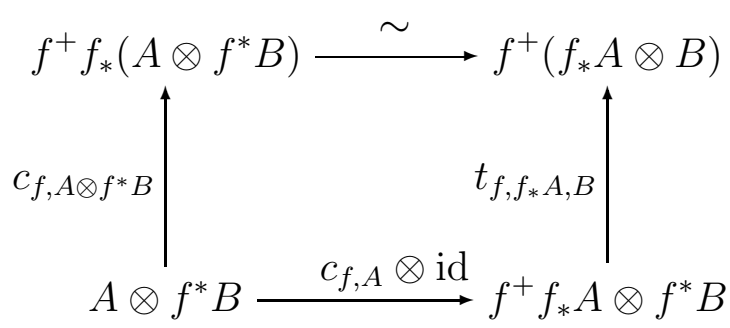


with the top horizontal arrow induced by the projection formula, is commutative. Applying this to $f=\delta, A=F$ and $g=p_{2}^{*} G$ we deduce the commutativity of the left square in the diagram

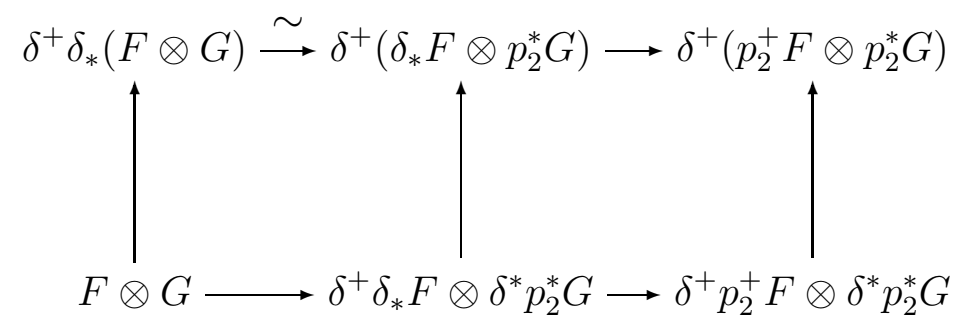

Note that the second square is commutative by the functoriality of the map $\delta^{+} A \otimes \delta^{*} B \rightarrow$ $\delta^{+}(A \otimes B)$ in $A$. It follows that the composition of the first three arrows in (5.5) is equal to the map

$$
F \otimes G \simeq \delta^{+} p_{2}^{+} F \otimes \delta^{*} p_{2}^{*} G \rightarrow \delta^{+}\left(p_{2}^{+} F \otimes p_{2}^{*} G\right) .
$$

Next, for composable arrows $f$ and $g$ we have a commutative diagram

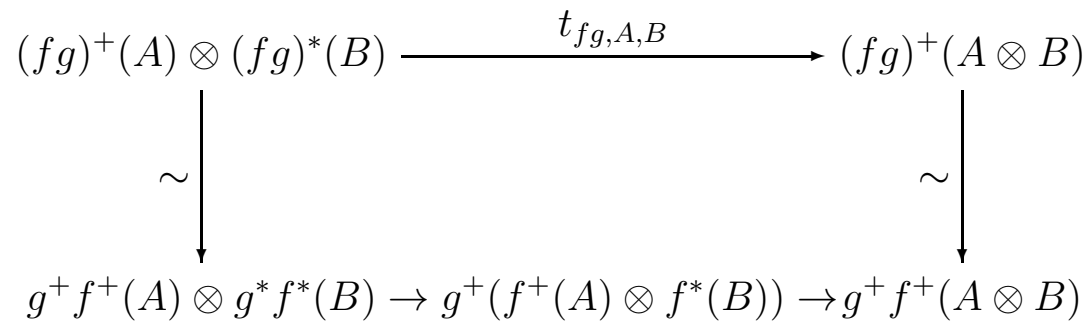

Applying this for $f=p_{2}, g=\delta, A=F$ and $B=G$ we deduce that the composition of (5.6)) with the last arrow in (5.5),

$$
\delta^{+}\left(p_{2}^{+} F \otimes p_{2}^{*} G\right) \stackrel{\sim}{\longrightarrow} \delta^{+} p_{2}^{+}(F \otimes G) \simeq F \otimes G,
$$

is the identity map of $F \otimes G$.

The case of matrix factorization reduces to the case of ( $\mathbb{G}_{m}$-equivariant) sheaves using the equivalences with the $\left(\mathbb{G}_{m}\right.$-equivariant) singularity categories. Namely, let $Z_{0} \subset Z$ be the hypersurface of zeros of $W, Y_{0}=f^{-1}\left(Z_{0}\right)$. Note that the hypersurface of zeros of $\pi^{*} W$ is

$$
\pi^{-1}\left(Z_{0}\right)=Y_{0} \times{ }_{Z_{0}} Y_{0}
$$

Now $G$ corresponds to an object of $D_{\mathrm{Sg}, \mathbb{G}_{m}}\left(Y_{0}\right)$, while the commutative diagram lives in the category of matrix factorizations of $\pi^{*} W$, which is equivalent to the category $D_{\mathrm{Sg}, \mathbb{G}_{m}}\left(Y_{0} \times Z_{0}\right.$ $\left.Y_{0}\right)$. Now we observe that the functors $p_{2}^{*}$ and $p_{2}^{+}$from matrix factorizations of $f^{*} W$ to those of $\pi^{*} W$ correspond to the similar functors

$$
p_{2}^{*}, p_{2}^{+}: D_{\mathrm{Sg}, \mathbb{G}_{m}}\left(Y_{0}, f^{*} W\right) \rightarrow D_{\mathrm{Sg}, \mathbb{G}_{m}}\left(Y_{0} \times_{Z_{0}} Y_{0}\right)
$$

The operation of tensoring a matrix factorization with an object $P$ of the perfect derived category corresponds for the category of singularity to the operation of tensoring with the 
restriction of $P$ to the zero locus of the potential (we apply this for $f^{*} W$ and for $\pi^{*} W$ ). Finally, we use the fact that

$$
\left.\delta_{*} F\right|_{\pi 1\left(Z_{0}\right)} \simeq \bar{\delta}_{*}\left(\left.F\right|_{Y_{0}}\right),
$$

where $\bar{\delta}: Y_{0} \rightarrow Y_{0} \times{ }_{Z_{0}} Y_{0}$ is the diagonal, that follows from the base change formula.

(iii) Using the definition this reduces to checking the equality of the maps

$$
\begin{gathered}
\mathcal{O}_{Y} \stackrel{\sim}{\longrightarrow} \delta^{+} p_{1}^{+} f^{+} \mathcal{O}_{Z} \quad \text { and } \\
\mathcal{O}_{Y} \stackrel{\sim}{\longrightarrow} \delta^{+} p_{2}^{+} f^{+} \mathcal{O}_{Z}
\end{gathered}
$$

under the identification $\pi^{+} \mathcal{O}_{Z} \simeq p_{1}^{+} f^{+} \mathcal{O}_{Z} \simeq p_{2}^{+} f^{+} \mathcal{O}_{Z}$. This reduces to the commutativity of the diagram with standard isomorphisms

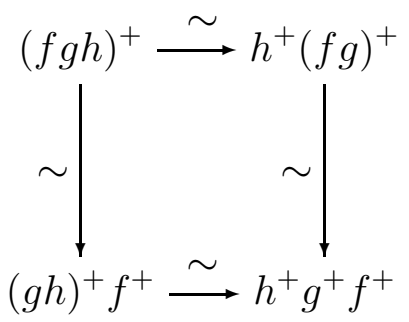

applied to the triples of morphisms $\left(f, p_{1}, \delta\right)$ and $\left(f, p_{2}, \delta\right)$.

Next, we assume that our map $f$ fits into a diagram

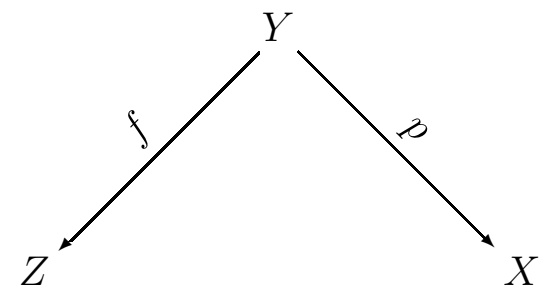

and that we are given $P \in D^{b}(Y)$, with proper support. Let us set $Q=P^{\vee} \otimes \mathcal{D}_{f}$. Note that we have a natural isomorphism $p_{2}^{+} \mathcal{O}_{Y} \simeq p_{1}^{*} \mathcal{D}_{f}$. Hence, we obtain a natural map on $Y \times_{Z} Y$,

$$
\begin{aligned}
& \widetilde{\varphi}: \delta_{*} \mathcal{O}_{Y} \rightarrow \delta_{*}\left(P^{\vee} \otimes P\right) \simeq \delta_{*} \mathcal{O}_{Y} \otimes p_{1}^{*} P^{\vee} \otimes p_{2}^{*} P \stackrel{\alpha_{\mathcal{O}} \otimes \mathrm{id}}{\longrightarrow} p_{1}^{*} \mathcal{D}_{f} \otimes p_{1}^{*} P^{\vee} \otimes p_{2}^{*} P \simeq \\
& p_{1}^{*}\left(\mathcal{D}_{f} \otimes P^{\vee}\right) \otimes p_{2}^{*} P=p_{1}^{*} Q \otimes p_{2}^{*} P,
\end{aligned}
$$

where $\delta: Y \rightarrow Y \times_{Z} Y$ is the relative diagonal and $\alpha_{\mathcal{O}}$ is the map (5.4). Let $p_{X X}: Y \times_{Z} Y \rightarrow$ $X \times X$ be the map with the components $\left(p p_{1}, p p_{2}\right)$. Applying $p_{X X, *}$ to the above map and using the natural map $\mathcal{O}_{X} \rightarrow p_{*} \mathcal{O}_{Y}$ we get a canonical morphism

$$
\varphi: \Delta_{*} \mathcal{O}_{X} \rightarrow \Delta_{*} p_{*} \mathcal{O}_{Y} \simeq p_{X X, *} \delta_{*} \mathcal{O}_{Y} \rightarrow p_{X X, *}\left(p_{1}^{*} Q \otimes p_{2}^{*} P\right),
$$

where $\Delta=\Delta_{X}: X \rightarrow X \times X$ is the diagonal map.

We also consider an analogous construction for matrix factorizations in the situation $(\star)$, where $P$ is an object of $\operatorname{MF}_{\mathbb{G}_{m}}\left(Y,-f^{*} W\right)$ with proper support. We then view $Q$ as an object 
of $\mathrm{MF}_{\mathbb{G}_{m}}\left(Y, f^{*} W\right)$, and the analog of the map (5.7) can be constructed in $\operatorname{MF}_{\mathbb{G}_{m}, d}\left(Y \times_{Z} Y, 0\right)$. Assuming in addition that $X$ is smooth (so we can regard $\Delta_{*} \mathcal{O}_{X}$ as a perfect complex on $X \times X)$, we get an analog of the map $\varphi$ in $\mathrm{MF}_{\mathbb{G}_{m}, d}(X \times X, 0)$. Recall that in this situation we have functors

$$
\widetilde{\Phi}_{P}: \operatorname{MF}_{\mathbb{G}_{m}}(Z, W) \rightarrow \mathrm{MF}_{\mathbb{G}_{m}, d}(X, 0), \widetilde{\Psi}_{Q}: \operatorname{MF}_{\mathbb{G}_{m}, d}(X, 0) \rightarrow \operatorname{MF}_{\mathbb{G}_{m}}(Z, W)
$$

(see Sec. 2.6). In the case of sheaves we also denote by $\widetilde{\Phi}_{P}$ and $\widetilde{\Psi}_{Q}$ the similar Fourier-Mukai functors between $D^{b}(Z)$ and $D^{b}(X)$.

The main result of this Appendix is the following compatibility (needed for the proof of Proposition 2.6.2).

Proposition 5.0.3. In the two situations described above (with sheaves and with matrix factorizations) the map

$$
\operatorname{Hom}\left(\widetilde{\Psi}_{Q}(E), F\right) \rightarrow \operatorname{Hom}\left(E, \widetilde{\Phi}_{P}(F)\right)
$$

obtained via (2.2) from the natural transformation $\mathrm{Id} \rightarrow \widetilde{\Phi}_{P} \circ \widetilde{\Psi}_{Q}$, induced by (15.8), is equal to the composition

$$
\begin{aligned}
& \operatorname{Hom}\left(f_{*}\left(p^{*} E \otimes Q\right), F\right) \stackrel{\sim}{\longrightarrow} \operatorname{Hom}\left(p^{*} E \otimes Q, f^{*} F \otimes \mathcal{D}_{f}\right) \simeq \operatorname{Hom}\left(p^{*} E, f^{*} F \otimes P\right) \stackrel{\sim}{\longrightarrow} \\
& \operatorname{Hom}\left(E, p_{*}\left(f^{*} F \otimes P\right)\right) .
\end{aligned}
$$

Proof. By definition, we have to prove that the composition (5.9) is equal to the map

$$
\operatorname{Hom}\left(\widetilde{\Psi}_{Q}(E), F\right) \stackrel{\widetilde{\Phi}_{P}}{\longrightarrow} \operatorname{Hom}\left(\widetilde{\Phi}_{P} \widetilde{\Psi}_{Q}(E), \widetilde{\Phi}_{P}(F)\right) \rightarrow \operatorname{Hom}\left(E, \widetilde{\Phi}_{P}(F)\right),
$$

where the second arrow is induced by (5.8). Unraveling this leads to the following composition

$$
\begin{aligned}
& \operatorname{Hom}\left(f_{*}\left(p^{*} E \otimes Q\right), F\right) \stackrel{(1)}{\longrightarrow} \operatorname{Hom}\left(p_{2 *} p_{1}^{*}\left(p^{*} E \otimes Q\right), f^{*} F\right) \stackrel{(2)}{\longrightarrow} \\
& \operatorname{Hom}\left(p^{*} E \otimes p_{2 *}\left(p_{1}^{*} Q \otimes p_{2}^{*} P\right), f^{*} F \otimes P\right) \stackrel{(3)}{\longrightarrow} \operatorname{Hom}\left(E, p_{*}\left(f^{*} F \otimes P\right)\right),
\end{aligned}
$$

with the intermediate maps given by

$$
\text { (1) } \operatorname{Hom}\left(f_{*}\left(p^{*} E \otimes Q\right), F\right) \stackrel{f^{*}}{\longrightarrow} \operatorname{Hom}\left(f^{*} f_{*}\left(p^{*} E \otimes Q\right), f^{*} F\right) \stackrel{\sim}{\longrightarrow} \operatorname{Hom}\left(p_{2 *} p_{1}^{*}\left(p^{*} E \otimes Q\right), f^{*} F\right) \text {, }
$$

where the second arrow is induced by the base change isomorphism $f^{*} f_{*} \stackrel{\sim}{\longrightarrow} p_{2 *} p_{1}^{*}$;

$$
\begin{aligned}
& (2): \operatorname{Hom}\left(p_{2 *} p_{1}^{*}\left(p^{*} E \otimes Q\right), f^{*} F\right) \stackrel{\otimes P}{\longrightarrow} \operatorname{Hom}\left(p_{2 *} p_{1}^{*}\left(p^{*} E \otimes Q\right) \otimes P, f^{*} F \otimes P\right) \simeq \\
& \operatorname{Hom}\left(p_{2 *}\left(p_{1}^{*} p^{*} E \otimes p_{1}^{*} Q \otimes p_{2}^{*} P\right), f^{*} F \otimes P\right) ;
\end{aligned}
$$

and

$$
\begin{aligned}
& (3): \operatorname{Hom}\left(p_{2 *}\left(p_{1}^{*} p^{*} E \otimes p_{1}^{*} Q \otimes p_{2}^{*} P\right), f^{*} F \otimes P\right) \stackrel{p_{*}}{\longrightarrow} \\
& \operatorname{Hom}\left(p_{2 *}^{X} p_{X X, *}\left(p_{1}^{*} p^{*} E \otimes p_{1}^{*} Q \otimes p_{2}^{*} P\right), p_{*}\left(f^{*} F \otimes P\right)\right) \simeq \\
& \operatorname{Hom}\left(p_{2 *}^{X}\left(p_{1}^{X, *} E \otimes p_{X X, *}\left(p_{1}^{*} Q \otimes p_{2}^{*} P\right)\right), p_{*}\left(f^{*} F \otimes P\right)\right) \stackrel{\varphi}{\longrightarrow} \\
& \operatorname{Hom}\left(p_{2 *}^{X}\left(p_{1}^{X, *} E \otimes \Delta_{*} \mathcal{O}_{X}\right), p_{*}\left(f^{*} F \otimes P\right)\right) \simeq \operatorname{Hom}\left(E, p_{*}\left(f^{*} F \otimes P\right)\right),
\end{aligned}
$$


where $p_{i}^{X}: X \times X \rightarrow X$, for $i=1,2$, are the projections. Here we used the natural identifications $p_{*} p_{2 *} \simeq p_{2 *}^{X} p_{X X, *}, p_{1}^{*} p^{*} \simeq p_{X X}^{*} p_{1}^{X, *}$ and the projection formula for $p_{X X}$.

Let us set for brevity $R:=p_{1}^{*} Q \otimes p_{2}^{*} P$ and $\widetilde{F}:=f^{*} F \otimes P$. We claim that the map

$$
\left(3^{\prime}\right): \operatorname{Hom}\left(p_{2 *}\left(p_{1}^{*} p^{*} E \otimes R\right), \widetilde{F}\right) \rightarrow \operatorname{Hom}\left(p^{*} E, \widetilde{F}\right),
$$

that corresponds to $(3)$ under the identification $\operatorname{Hom}\left(E, p_{*}\left(f^{*} F \otimes P\right)\right) \simeq \operatorname{Hom}\left(p^{*} E, f^{*} F \otimes P\right)$, is given simply by the composition

$\operatorname{Hom}\left(p_{2 *}\left(p_{1}^{*} p^{*} E \otimes R\right), \widetilde{F}\right) \stackrel{\widetilde{\varphi}}{\longrightarrow} \operatorname{Hom}\left(p_{2 *}\left(p_{1}^{*} p^{*} E \otimes \delta_{*} \mathcal{O}_{Y}\right), \widetilde{F}\right) \simeq \operatorname{Hom}\left(p_{2 *} \delta_{*}\left(p^{*} E\right), \widetilde{F}\right) \simeq \operatorname{Hom}\left(p^{*} E, \widetilde{F}\right)$, where the first arrow is induced by (5.7). Indeed, first, using the definition of $\varphi$ we can rewrite (3) as the composition

$$
\begin{aligned}
& \operatorname{Hom}\left(p_{2 *}\left(p_{1}^{*} p^{*} E \otimes R\right), \widetilde{F}\right) \stackrel{p_{*}}{\longrightarrow} \operatorname{Hom}\left(p_{2 *}^{X} p_{X X, *}\left(p_{1}^{*} p^{*} E \otimes R\right), p_{*} \widetilde{F}\right) \stackrel{\widetilde{\varphi}}{\longrightarrow} \\
& \operatorname{Hom}\left(p_{2 *}^{X} p_{X X, *}\left(p_{1}^{*} p^{*} E \otimes \delta_{*} \mathcal{O}_{Y}\right), p_{*} \widetilde{F}\right) \simeq \operatorname{Hom}\left(p_{2 *}^{X}\left(p_{1}^{X, *} E \otimes p_{X X, *} \delta_{*} \mathcal{O}_{Y}\right), p_{*} \widetilde{F}\right) \rightarrow \\
& \operatorname{Hom}\left(p_{2 *}^{X}\left(p_{1}^{X, *} E \otimes \Delta_{*} \mathcal{O}_{X}\right), p_{*} \widetilde{F}\right) \simeq \operatorname{Hom}\left(E, p_{*} \widetilde{F}\right) .
\end{aligned}
$$

Now we observe that there is a commutative diagram

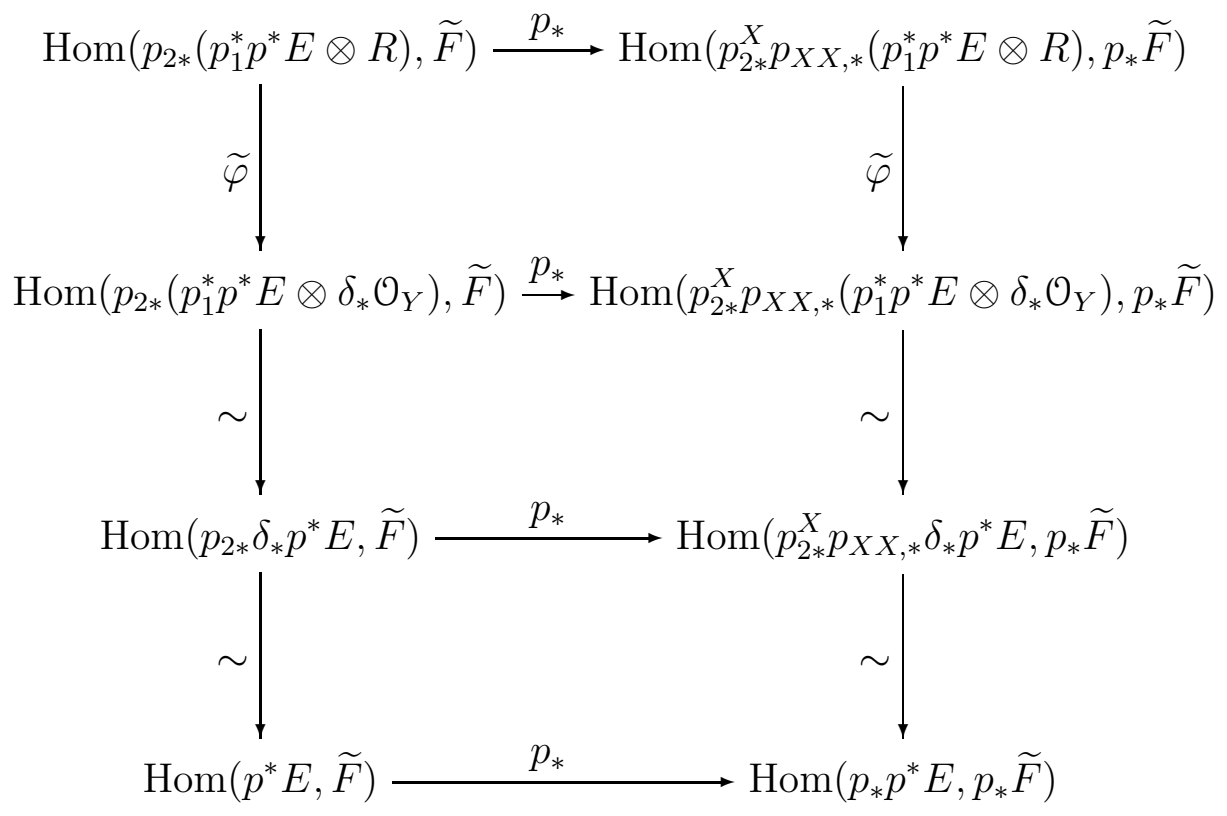

Hence, our claim about the map $\left(3^{\prime}\right)$ follows from the commutativity of the diagram

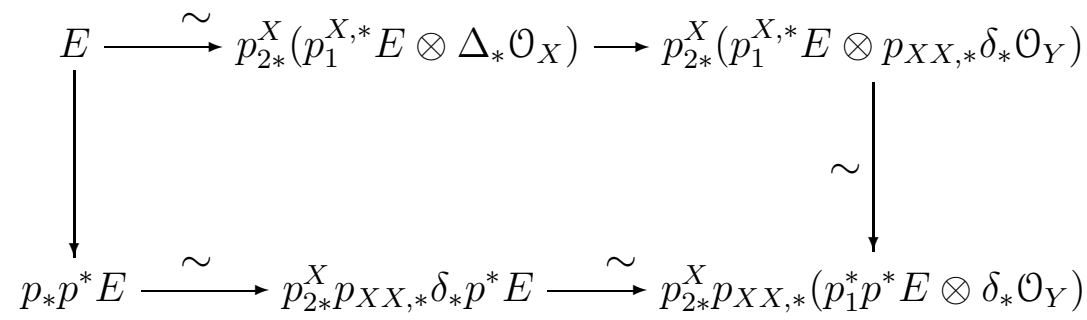


which is easy to check.

Our description of $\left(3^{\prime}\right)$ implies that we have a commutative diagram

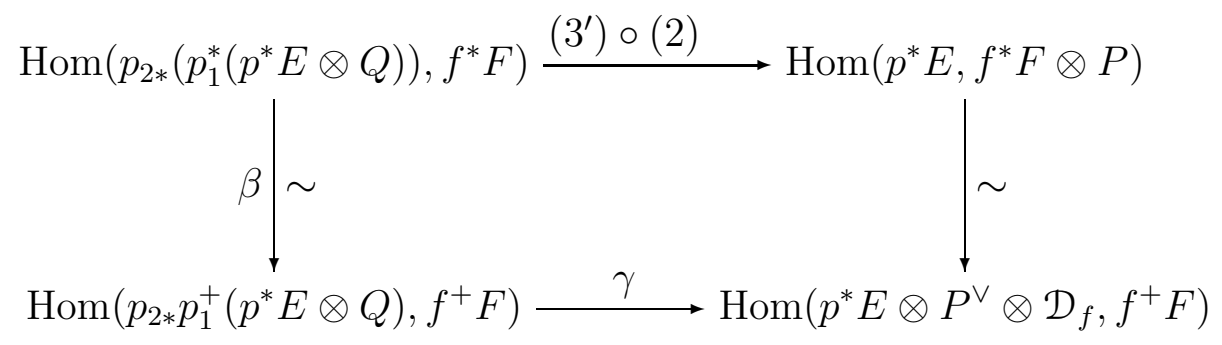

Here $\beta$ is the composition of the natural isomorphisms

$$
\begin{aligned}
& \beta: \operatorname{Hom}\left(p_{2 *} p_{1}^{*}\left(p^{*} E \otimes Q\right), f^{*} F\right) \stackrel{\sim}{\longrightarrow} \operatorname{Hom}\left(p_{2 *} p_{1}^{*}\left(p^{*} E \otimes Q\right) \otimes \mathcal{D}_{f}, f^{*} F \otimes \mathcal{D}_{f}\right) \stackrel{\sim}{\longrightarrow} \\
& \operatorname{Hom}\left(p_{2 *} p_{1}^{+}\left(p^{*} E \otimes Q\right), f^{+} F\right),
\end{aligned}
$$

where in the second isomorphism we use the identification $p_{1}^{+} \mathcal{O}_{Y} \simeq p_{2}^{*} \mathcal{D}_{f}$, and $\gamma$ is the composition

$$
\gamma: \operatorname{Hom}\left(p_{2 *} p_{1}^{+}\left(p^{*} E \otimes Q\right), f^{+} F\right) \rightarrow \operatorname{Hom}\left(p_{2 *}\left(p_{1}^{+}\left(p^{*} E \otimes Q\right) \otimes p_{2}^{*} P \otimes p_{2}^{*} P^{\vee}\right), f^{+} F\right) \rightarrow
$$$$
\operatorname{Hom}\left(p^{*} E \otimes P^{\vee} \otimes \mathcal{D}_{f}, f^{+} F\right) \text {, }
$$

where the first arrow is induced by the evaluation map $e v_{P}: P \otimes P^{\vee} \rightarrow \mathcal{O}_{Y}$, while the second arrow is induced by the map

$$
\begin{aligned}
& p^{*} E \otimes P^{\vee} \otimes \mathcal{D}_{f} \simeq p_{2 *}\left(p_{1}^{*} p^{*} E \otimes \delta_{*}\left(P^{\vee} \otimes \mathcal{D}_{f}\right)\right) \stackrel{p_{2 *}(\mathrm{id} \otimes \epsilon)}{\longrightarrow} p_{2 *}\left(p_{1}^{*} p^{*} E \otimes p_{1}^{+} Q \otimes p_{2}^{*} P \otimes p_{2}^{*} P^{\vee}\right) \simeq \\
& p_{2 *}\left(p_{1}^{+}\left(p^{*} E \otimes Q\right) \otimes p_{2}^{*} P \otimes p_{2}^{*} P^{\vee}\right),
\end{aligned}
$$

where $\epsilon$ is the composition

$\epsilon: \delta_{*}\left(P^{\vee} \otimes \mathcal{D}_{f}\right) \simeq \delta_{*} \mathcal{O}_{Y} \otimes p_{2}^{*} P^{\vee} \otimes p_{2}^{*} \mathcal{D}_{f} \stackrel{\widetilde{\varphi} \otimes \mathrm{id}}{\longrightarrow} p_{1}^{*} Q \otimes p_{2}^{*} P \otimes p_{2}^{*} P^{\vee} \otimes p_{2}^{*} \mathcal{D}_{f} \simeq p_{1}^{+} Q \otimes p_{2}^{*} P \otimes p_{2}^{*} P^{\vee}$.

Next, let us consider the composed map $\left(1^{\prime}\right): \operatorname{Hom}\left(f_{*}\left(p^{*} E \otimes Q\right), F\right) \stackrel{(1)}{\longrightarrow} \operatorname{Hom}\left(p_{2 *} p_{1}^{*}\left(p^{*} E \otimes Q\right), f^{*} F\right) \stackrel{\beta}{\longrightarrow} \operatorname{Hom}\left(p_{2 *} p_{1}^{+}\left(p^{*} E \otimes Q\right), f^{+} F\right)$. Applying Lemma 5.0.2(i) to the object $p^{*} E \otimes Q$ we get that the composition

$$
p^{*} E \otimes Q \simeq p_{2 *} \delta_{*}\left(p^{*} E \otimes Q\right) \stackrel{p_{2 *}\left(\alpha_{p^{*} E \otimes Q}\right)}{\longrightarrow} p_{2 *} p_{1}^{+}\left(p^{*} E \otimes Q\right) \rightarrow f^{+} f_{*}\left(p^{*} E \otimes Q\right)
$$

is just the map (5.1). This implies the commutativity of the following triangle

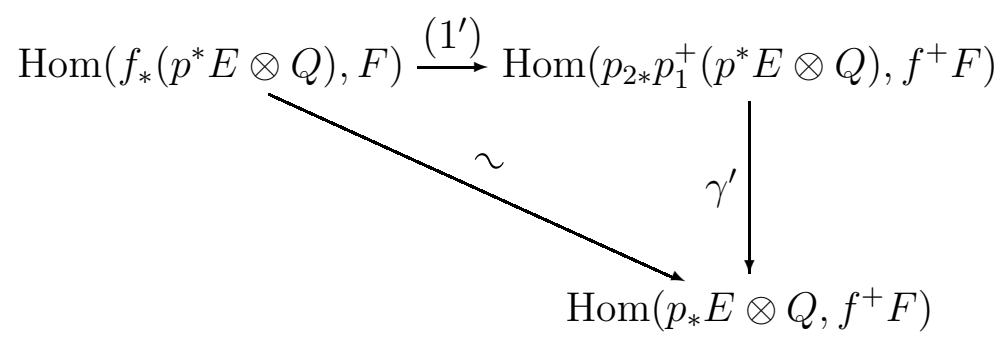


where the diagonal is the adjunction isomorphism and $\gamma^{\prime}$ is induced by the map

$$
p^{*} E \otimes Q \simeq p_{2 *} \delta_{*}\left(p^{*} E \otimes Q\right) \stackrel{p_{2 *}\left(\alpha_{p^{*} E \otimes Q}^{\prime}\right)}{\longrightarrow} p_{2 *} p_{1}^{+}\left(p^{*} E \otimes Q\right) .
$$

Comparing the diagrams (5.10) and (5.12) with the definition of the map (5.9), we see that our assertion would follow from the equality $\gamma^{\prime}=\gamma$, which in turn would be implied by the commutativity of the diagram

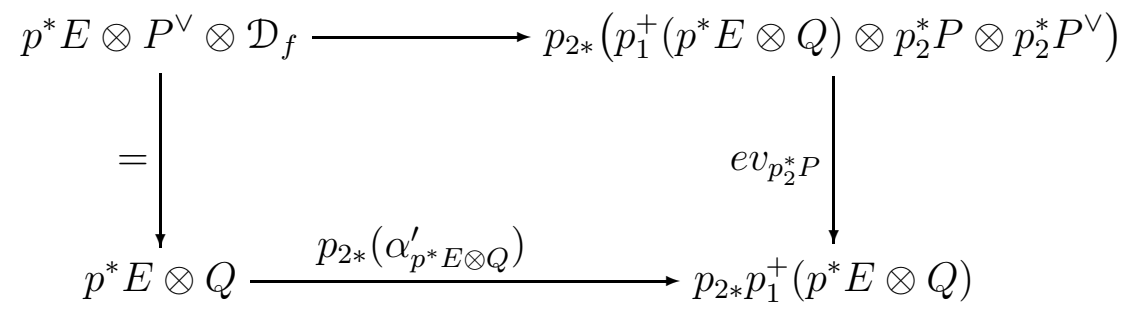

where the top horizontal arrow is (5.11). Note that this diagram is obtained by applying the functor $p_{2 *}$ to the diagram

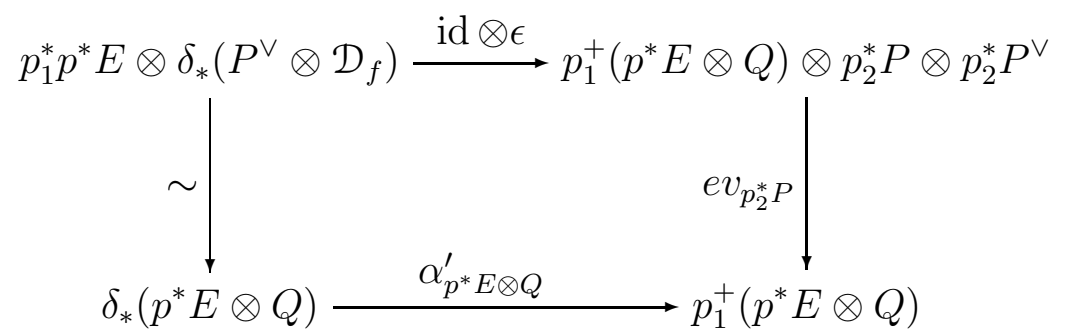

Unraveling the definition of $\epsilon$ and using Lemma 5.0.2(ii), we see that the latter diagram is obtained by tensoring with $p_{1}^{*} p^{*} E$ from the diagram

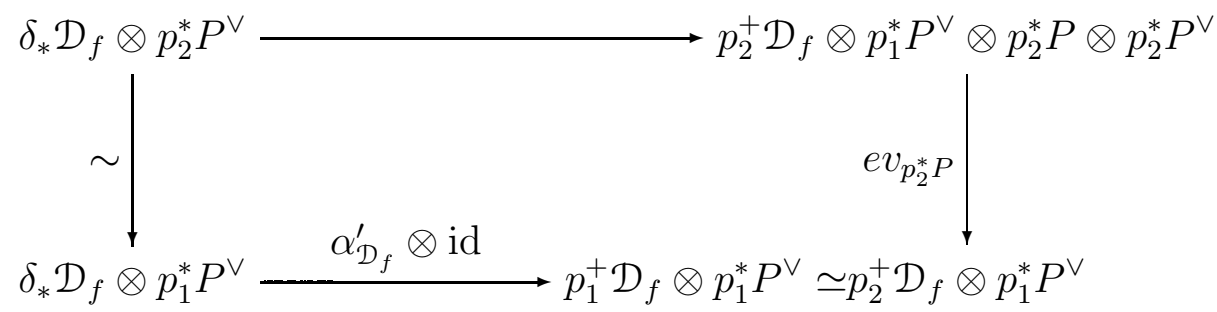

where the top arrow is the composition

$$
\delta_{*} \mathcal{D}_{f} \otimes p_{2}^{*} P^{\vee} \rightarrow \delta_{*} \mathcal{D}_{f} \otimes p_{2}^{*} P^{\vee} \otimes p_{2}^{*} P \otimes p_{1}^{*} P^{\vee} \stackrel{\alpha_{\mathcal{D}_{f}} \otimes \mathrm{id}}{\longrightarrow} p_{2}^{+} \mathcal{D}_{f} \otimes p_{2}^{*} P^{\vee} \otimes p_{2}^{*} P \otimes p_{1}^{*} P^{\vee}
$$

Thus, it remains to prove the commutativity of (5.13). Using the commutative diagram

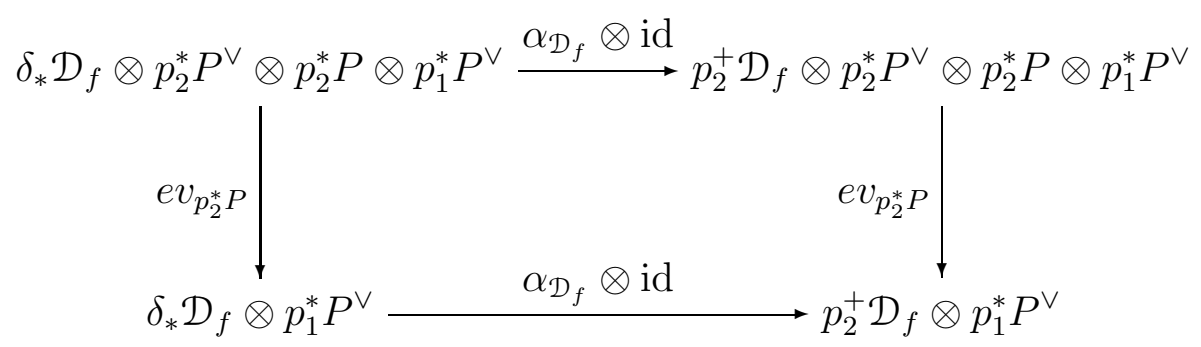


we can rewrite the composition of the top arrow with the right vertical arrow in (5.13) as the map

$$
\delta_{*} \mathcal{D}_{f} \otimes p_{2}^{*} P^{\vee} \rightarrow \delta_{*} \mathcal{D}_{f} \otimes p_{2}^{*} P^{\vee} \otimes p_{2}^{*} P \otimes p_{1}^{*} P^{\vee} \stackrel{e v_{p_{2}^{*} P}}{\longrightarrow} \delta_{*} \mathcal{D}_{f} \otimes p_{1}^{*} P^{\vee} \stackrel{\alpha_{\mathcal{D}_{f}} \otimes \mathrm{id}}{\longrightarrow} p_{2}^{+} \mathcal{D}_{f} \otimes p_{1}^{*} P^{\vee}
$$

Here the composition of the first two arrows coincides with the left vertical arrow in (5.13), so we get the commutativity of the diagram like (5.13) but with $\alpha_{\mathcal{D}_{f}}$ instead of $\alpha_{\mathcal{D}_{f}}^{\prime}$ in the bottom arrow. It remains to recall that $\alpha_{\mathcal{D}_{f}}=\alpha_{\mathcal{D}_{f}}^{\prime}$ by Lemma [5.0.2(iii).

\section{References}

[1] M. Atiyah, Complex analytic connections in fibre bundles, Trans. AMS 85 (1957), 181207.

[2] M. Ballard, D. Deliu, D. Favero, M. U. Isik, L. Katzarkov, Resolutions in factorization categories, Adv. Math. 295 (2016), 195-249.

[3] D. Bergh, V.A. Lunts, O. M. Schnürer, Geometricity for derived categories of algebraic stacks, arXiv:1601.04465.

[4] A. Blanc, Topological K-theory of complex noncommutative spaces, Compos. Math. 152 (2016), no. 3, 489-555.

[5] A. Bondal, M. Kapranov, Representable functors, Serre functors, and reconstructions, Math. USSR-Izv. 35 (1990), no. 3, 519-541.

[6] A. Caldararu, The Mukai pairing. II. The Hochschild-Kostant-Rosenberg isomorphism, Adv. Math. 194 (2005) 34-66.

[7] A. Caldararu, J.Tu, Curved $A_{\infty}$-algebras and Landau-Ginzburg models, New York J. Math. 19 (2013), 305-342.

[8] A. Caldararu, S. Willerton, The Mukai Paring I: a categorical approach, New York J. Math. 16 (2010), 61-98.

[9] H.-L. Chang, J. Li, W.-P. Li, Witten's top Chern class via cosection localization, Invent. Math. 200 (2015), no. 3, 1015-1063.

[10] A. Chiodo, Witten's top Chern class via K-theory, J. Algebraic Geom. 15 (2006), no. 4, 681-707.

[11] A. Chiodo, Y. Ruan, LG/CY correspondence: the state space isomorphism, Adv. Math. 227 (2011), no. 6, 2157-2188.

[12] A. Chiodo, H. Iritani, Y.Ruan, Landau-Ginzburg/Calabi-Yau correspondence, global mirror symmetry and Orlov equivalence, Publ. Math. IHES 119 (2014), 127-216. 
[13] P. Deligne, Hodge cycles on abelian varieties, in Hodge cycles, motives, and Shimura varieties, LNM 900, Springer-Verlag, Berlin-New York, 1982.

[14] T. Dyckerhoff, Compact generators in categories of matrix factorizations, Duke Math. J. 159 (2011), 223-274.

[15] A. Efimov, L. Positselski, Coherent analogues of matrix factorizations and relative singularity categories, Algebra Number Theory 9 (2015), no. 5, 1159-1292.

[16] H. Fan, T. Jarvis, Y. Ruan, The Witten equation, mirror symmetry and quantum singularity theory, Ann. of Math. (2) 178 (2013), 1-106.

[17] J. Guéré, A Landau-Ginzburg mirror theorem without concavity, arXiv:1307.5070.

[18] R. Hartshorne, Residues and Duality, Lecture Notes in Math. 20, Springer-Verlag, Berlin, 1966.

[19] M. Kontsevich, XI Solomon Lefschetz Memorial Lecture Series: Hodge structures in noncommutative geometry (Notes by Ernesto Lupercio), in Non-commutative geometry in mathematics and physics, 1-21, AMS, Providence, RI, 2008.

[20] M. Kontsevich, Yu. I. Manin, Gromov-Witten classes, quantum cohomology, and enumerative geometry, Commun. Math. Phys. 164 (1994), 525-562.

[21] A. Kresch, On the geometry of Deligne-Mumford stacks, in Algebraic geometry (Seattle 2005), Part 1, 259-271, Amer. Math. Soc., Providence, RI, 2009.

[22] A. Kresch, A. Vistoli, On coverings of Deligne-Mumford stacks and surjectivity of the Brauer map, Bull. London Math. Soc. 36 (2004), no. 2, 188-192.

[23] A. Kuznetsov, Hochschild homology and semiorthogonal decompositions, arXiv:0904.4330.

[24] A. Kuznetsov, Base change for semiorthogonal decompositions, Compos. Math. 147 (2011), 852-876.

[25] K. Lin, D. Pomerleano, Global matrix factorizations, Math. Res. Lett. 20 (2013), no. 1, 91-106.

[26] E. Macrí, P.Stellari, Infinitesimal derived Torelli theorem for K3 surfaces, IMRN 17 (2009), 3190-3220.

[27] W. F. Moore, G. Piepmeyer, S. Spiroff, M. E. Walker, Hochster's theta invariant and the Hodge-Riemann bilinear relations, Adv. Math. 226 (2011), 1692-1714.

[28] D. Orlov, Triangulated categories of singularities and D-branes in Landau-Ginzburg models, Proc. Steklov Inst. Math. 2004, no. 3 (246), 227-248. 
[29] D. Orlov, Derived categories of coherent sheaves and triangulated categories of singularities, in Algebra, arithmetic, and geometry: in honor of Yu. I. Manin, Vol. II, 503-531, Birkhauser, Boston, MA, 2009.

[30] D. Orlov, Matrix factorizations for nonaffine LG-models, Math. Ann. 353 (2012), 95108.

[31] D. Orlov, Smooth and proper noncommutative schemes and gluing of DG categories, arXiv:1402.7364.

[32] A. Polishchuk, Lefschetz type formulas for dg-categories, Selecta Math. 20 (2014), 885928.

[33] A. Polishchuk and A. Vaintrob, Algebraic construction of Witten's top Chern class, in Advances in algebraic geometry motivated by physics (Lowell, MA, 2000), 229-249, Amer. Math. Soc., Providence, RI, 2001.

[34] A. Polishchuk, A. Vaintrob, Chern characters and Hirzebruch-Riemann-Roch formula for matrix factorizations, Duke Math. J. 161 (2012), 1863-1926.

[35] A. Polishchuk, A. Vaintrob, Matrix factorizations and singularity categories for stacks, Ann. Inst. Fourier 61 (2011), 2609-2642.

[36] A. Polishchuk, A. Vaintrob, Matrix factorizations and Cohomological Field Theories, J. Reine Angew. Math. 714 (2016), 1-122.

[37] A. C. Ramadoss, The relative Riemann-Roch theorem from Hochschild homology, New York J. Math. 14 (2008), 643-717.

[38] I. Shipman, A geometric approach to Orlov's theorem, Compos. Math. 148 (2012), no. 5, 13651389.

[39] D.Shklyarov, Hirzebruch-Riemann-Roch-type theorem for DG algebras, Proc. London Math. Soc. 106 (2013), 1-32.

[40] R. W. Thomason, Algebraic K-theory of group scheme actions, in Algebraic topology and algebraic K-theory (Princeton, N.J., 1983), 539-563, Princeton Univ. Press, 1987.

[41] B. Toën, The homotopy theory of dg-categories and derived Morita theory, Invent. Math. 167 (2007), 615-667.

[42] B. Toën, M. Vaquié, Moduli of objects in dg-categories, Ann. Sci. ENS (4) 40 (2007), $387-444$.

[43] R. O. Wells, Differential analysis on complex manifolds, Springer-Verlag, New York, 1980 .

Department of Mathematics, University of Oregon, Eugene, OR 97405 